\title{
Biallelic mutations in the ubiquitin ligase RFWD3 cause Fanconi anemia
}

\author{
Kerstin Knies, ${ }^{1}$ Shojiro Inano, ${ }^{2,3}$ María J. Ramírez, ${ }^{4,5}$ Masamichi Ishiai, ${ }^{2}$ Jordi Surrallés, $, 4,5,6$ Minoru Takata, ${ }^{2}$ and Detlev Schindler \\ 'Department of Human Genetics, Biozentrum, University of Wurzburg, Wurzburg, Germany. ${ }^{2}$ Laboratory of DNA Damage Signaling, Department of Late Effects Studies, Radiation Biology Center, \\ Kyoto University, Kyoto, Japan. ${ }^{3}$ Department of Hematology and Oncology, Graduate School of Medicine, Kyoto University, Kyoto, Japan. ${ }^{4}$ Department of Genetics and Microbiology, Universitat Autònoma \\ de Barcelona, Bellaterra (Barcelona), Spain. ${ }^{5}$ Center for Biomedical Network Research on Rare Diseases (CIBERER), Madrid, Spain. ${ }^{6}$ Cenetics Department, Hospital de Sant Pau, Barcelona, Spain.
}

\begin{abstract}
The WD40-containing E3 ubiquitin ligase RFWD3 has been recently linked to the repair of DNA damage by homologous recombination (HR). Here we have shown that an RFWD3 mutation within the WD40 domain is connected to the genetic disease Fanconi anemia (FA). An individual presented with congenital abnormalities characteristic of FA. Cells from the patient carrying the compound heterozygous mutations c.205_206dupCC and c.1916T>A in RFWD3 showed increased sensitivity to DNA interstrand cross-linking agents in terms of increased chromosomal breakage, reduced survival, and cell cycle arrest in $\mathrm{C}_{2}$ phase. The cellular phenotype was mirrored in genetically engineered human and avian cells by inactivation of RFWD3 or introduction of the patient-derived missense mutation, and the phenotype was rescued by expression of wild-type RFWD3 protein. HR was disrupted in RFWD3-mutant cells as a result of impaired relocation of mutant RFWD3 to chromatin and defective physical interaction with replication protein A. $R f w d 3$ knockout mice appear to have increased embryonic lethality, are subfertile, show ovarian and testicular atrophy, and have a reduced lifespan resembling that of other FA mouse models. Although RFWD3 mutations have thus far been detected in a single child with FA, we propose RFWD3 as an FA gene, FANCW, supported by cellular paradigm systems and an animal model.
\end{abstract}

\section{Introduction}

Safeguarding of the genome is essential for the suppression of oncogenesis and for stem cell maintenance in many organisms, and is warranted by DNA repair mechanisms. If they fail on a genetic basis, DNA repair disorders occur. Fanconi anemia (FA) is such a rare human genomic instability disease. While variability occurs, recurrent features include developmental anomalies and malformations, most commonly growth retardation, cutaneous pigment displacement, and radial ray defects. Other frequent aspects of FA comprise early-onset bone marrow failure and cancer predisposition, specifically for acute myelogenous leukemia and head and neck squamous cell carcinoma (1-4). FA results from faults in the FA/BRCA pathway for DNA interstrand cross-link (ICL) repair, in which proteins encoded by 21 FA genes reported thus far - designated as or aliased with the prefix FANC- and serial letters of the alphabet, FANCA to FANCV - and associated proteins interact (5-9). Germline inactivation of any one of the FA genes causes classical FA or variant (FA-like) disease lacking single clinical core features of FA. Common to all subtypes is cellular sensitivity to endogenously or exogenously produced ICLs, implicating spontaneous and induced chromosomal breakage, reduced cell survival, and arrest in $G_{2}$ phase of the cell cycle (2). The FA/ BRCA pathway orchestrates the action of other DNA repair proteins such as nucleases, translesion polymerases, and effectors of

Conflict of interest: The authors have declared that no conflict of interest exists.

Submitted: December 7, 2016; Accepted: May 16, 2017.

Reference information: / Clin Invest. 2017;127(8):3013-3027.

https://doi.org/10.1172/JCI92069. homologous recombination (HR), and interfaces with other DNA repair networks with which it shares components. New elements of the FA/BRCA pathway continue to be identified in ways that keep our understanding of this pathway evolving $(5,8-10)$.

Many of the FA proteins undergo secondary modification. Ubiquitination has become an important issue of ICL repair, including ubiquitin donors and acceptors (7). Ubiquitin transfer is facilitated by the coordinate action of 3 different types of enzymes, E1 for activation, E2 for conjugation, and E3 for ligation (11). The monoubiquitination of FANCD2 and FANCI by the E3 ligase FANCL and the E2 ligase UBE2T (also known as FANCT) are key steps of the FA/BRCA pathway (7). Compromised function of FANCD2, FANCI, FANCL, or FANCT due to defective ubiquitin transfer can abrogate the FA/BRCA pathway (9). In the opposite direction, the deubiquitinating enzyme complex USP1-UAF1 critically regulates ICL repair mediated by the FA/BRCA pathway through its deubiquitination activity toward FANCD2-Ub and FANCI-Ub. It has been recognized that the USP1-UAF1 complex also promotes HR (12). This occurs, at least in part, by UAF1stimulated RAD51-associated protein 1 (RAD51AP1) activity (13).

HR has emerged as the most important mechanism for the error-free repair of DNA double-strand lesions. Notably, certain proteins have been acknowledged as both late-acting components of the FA/BRCA pathway and actors in HR, including BRCA2, the partner and localizer of BRCA2 (PALB2), RAD51C, RAD51, BRCA1, and XRCC2, also known as FANCD1, -N, -O, -R, -S, and $-\mathrm{U}$, respectively. One of the major proteins early in $\mathrm{HR}$, replication protein A (RPA), not a known component of the FA/BRCA pathway, is polyubiquitinated by the RING-type E3 ubiquitin ligase 
RFWD3 on multiple lysine residues in response to DNA damage (14). Primarily described as an ATM and ATR phosphorylation substrate, RFWD3 accumulates at stalled replication forks as part of the DNA damage response (15). At these sites RFWD3 colocalizes with RPA and binds via its WD40 domain at the C-terminus to replication protein A2 (RPA2). RFWD3 functions in replication checkpoint signaling under replication stress and promotes replication fork restart after HR (14, 16-18). Recent work shows that RFWD3 ubiquitination of RPA is essential for productive HR (14). The departure of an RPA-single-stranded DNA complex and the recruitment of RAD51 alias FANCR, one of the most versatile factors in HR, advance the process $(19,20)$.

Here we report on a 12-year-old German individual with typical FA phenotype and compound heterozygous mutations in the E3 ubiquitin ligase RFWD3 (GenBank NM_018124.3). Based on our findings we conclude that biallelic germline mutations in $R F W D 3$ result in FA.

\section{Results}

Undefined subtype of a child with classical FA. The individual, referred to as 1143, offspring of unrelated parents of German descent, was born preterm (35 gestational weeks) and small for date $(1,850 \mathrm{~g})$. She presented with duodenal atresia, small midface and bilateral radial ray malformations including left radius hypoplasia, and absent thumb on both sides. Ultrasonography revealed enlarged lateral cerebral ventricles, a hypoplastic left kidney, and polysplenia. Growth retardation with length and weight below the third percentiles and microcephaly continued postnatally. Hypothalamic partial growth hormone deficiency gave cause for hormone replacement therapy starting as of 2 years, resulting in catch-up growth along the third percentiles for height and weight to date. MRI at age 2 years demonstrated rarefied periventricular white matter, a narrowed corpus callosum, downscaled anterior pituitary, ectopic position of the posterior pituitary in the hypothalamic region, and Chiari malformation type 1 . These changes did not cause neurological deficits or intellectual disability, such that she attends regular classes. This is consistent with the observation that minor brain malformations with little if any clinical manifestation are relatively frequent in FA patients (21). From age 10 her bone marrow was cytopenic with reduced hematopoiesis and increased fatty tissue. There were mild dysplastic features including dysmyelopoiesis (hypogranularity) and dysmegakaryopoiesis (abnormal lobulation), while erythropoiesis was slightly megaloblastoid. Myelodysplastic syndrome (MDS) was excluded on several occasions. Cytopenia is restricted to bone marrow; up to 12 years, her age at the time of writing, peripheral blood counts remained stable at a normal level. The clinical diagnosis of FA based on the indicated findings was confirmed by cellular hypersensitivity of cultured lymphocytes and fibroblasts to DNA-crosslinking agents (Supplemental Figure 1, A and B; supplemental material available online with this article; https://doi.org/10.1172/ JCI92069DS1). She was classified as belonging to a post-D2 FA subtype owing to intact cellular FANCD2 monoubiquitination (Supplemental Figure 1C). Proper RAD51 foci formation effectively ruled out groups FA-D1, -N and -O, -R, -S, and -U (Supplemental Figure 1D). Sanger sequencing of the remaining reported downstream FA genes failed to detect any mutation.
Whole exome sequencing identifies biallelic mutations of RFWD3. Assuming a yet unreported FA subgroup, whole exome sequencing (WES) was performed on fibroblast DNA. Statistical results of the data analysis are summarized in Supplemental Table 1. After exclusion of nonauthentic variants and formally inconsistent mutations, data mining left only 2 meaningful heterozygous mutations in the gene RFWD3 (Supplemental Table 2) that had been reported to be involved in the DNA damage response mediated by 553 and $\operatorname{RPA} 2(14,17,18)$. The 2 detected mutations, a 2-bp duplication resulting in premature termination of translation (c.205_206dupCC; p.L69Pfs ${ }^{\star} 12$ ) and a missense mutation (c.1916T>A; p.I639K) at a highly conserved position (Figure 1A), were confirmed by Sanger sequencing. They are located on different parental alleles and segregate in the family consistent with an autosomal recessive disorder (Figure 1B). Both variants are rare. They have not been reported in the 1000 Genomes Project (27,478 samples, February 2017) or in the Exome Aggregation Consortium (ExAC) database (60,706 samples, February 2017). Both variants were classified as probably damaging by several in silico prediction software tools. c.205_206dupCC (p.L69Pfs*12) is located in the N-terminal region of RFWD3, which is characterized by LQP-SSQ repeats of hitherto unknown function (18), whereas c.1916T $>$ A (p.I639K) is situated in the WD40 domain, which is responsible for RPA2 binding.

Wild-type RFWD3 reverses genomic instability and deficient HR of 1143 cells. While there is little doubt that c.205_206dupCC represents a functional null allele, reasonable concern was raised about the pathogenic nature of the missense variant p.I639K. To address this question, 1143 and control cultured fibroblasts were transduced with wild-type RFWD3 (WT-RFWD3) and control vectors. Susceptibility of cultured 1143 cells to specified chemical agents allowed for sensitivity testing. On cell cycle studies, FA-typical $\mathrm{G}_{2}$-phase accumulation following exposure to mitomycin C (MMC) or diepoxybutane (DEB) was restored to normal control levels in 1143 cells ectopically expressing WT-RFWD3, but not in mock-transduced 1143 cells (Supplemental Figure 1, E and F). Chromosomal breakage analysis showed accumulation of metaphases with $\geq 10$ breaks per nucleus including frequent radial figures (which reflect misdirected break repair by error-prone homology-directed pathways when HR is compromised) following exposure to MMC of nontransduced 1143 cells, or of those that were transduced with mock vector or vector containing the patient mutation, RFWD3-I639K cDNA (Figure 1C). In contrast, 1143 fibroblasts ectopically expressing WT-RFWD3 showed very few metaphases with high breakage rates. Moreover, survival of 1143 fibroblasts exposed to MMC was rescued after transduction with WT-RFWD3, whereas nontransduced 1143 cells, 1143 cells transduced with mock virus, or FA fibroblasts of subtype Q (FA-Q) showed significantly reduced survival in a dose-dependent manner (Figure 1D). 1143 fibroblasts were also hypersensitive to cisplatin (CDDP) to an extent that is comparable to that of FA-B or FA-Q fibroblasts (Figure 1E).

Like FA-D1 (biallelic BRCA2-mutant) cells with impaired HR, 1143 fibroblasts reveal sensitivity to the topoisomerase I inhibitor camptothecin. Decreased survival compared with non-FA control was rescued when 1143 cells were transduced with WT-RFWD3 (Figure 1F). Lastly, 1143 fibroblasts and those transduced with 
A

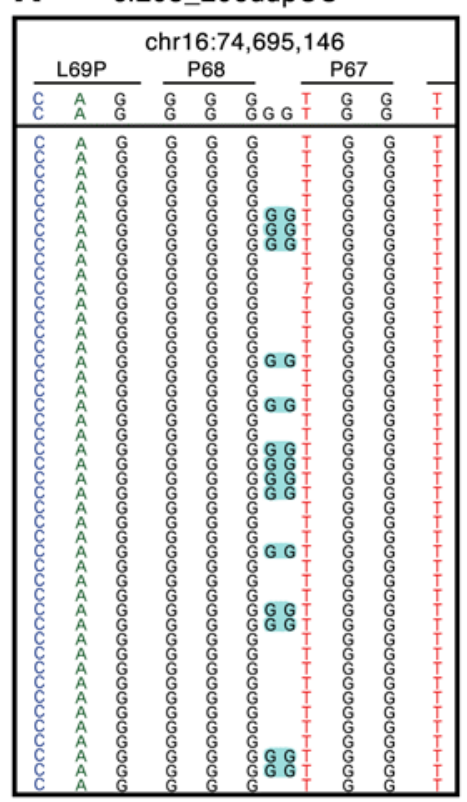

C

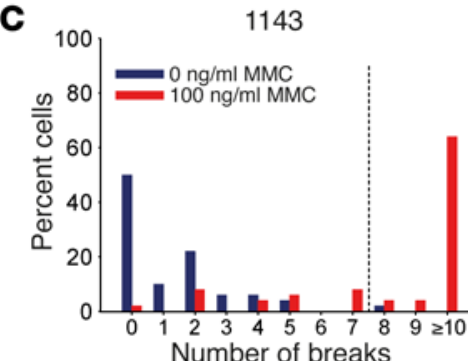

c. $1916 \mathrm{~T}>\mathrm{A}$

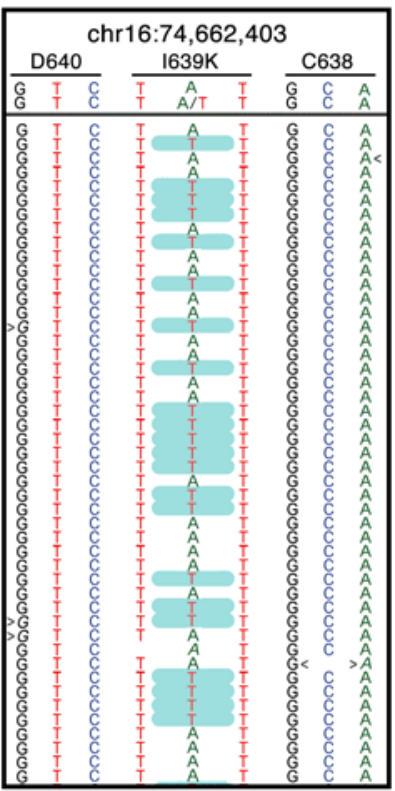

B
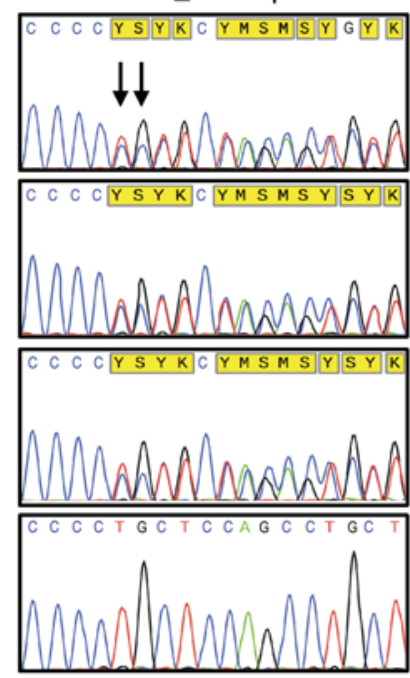

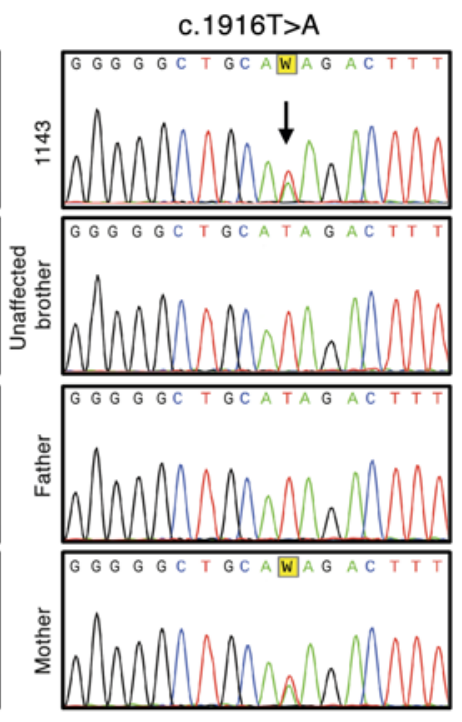

$1143+$ mock
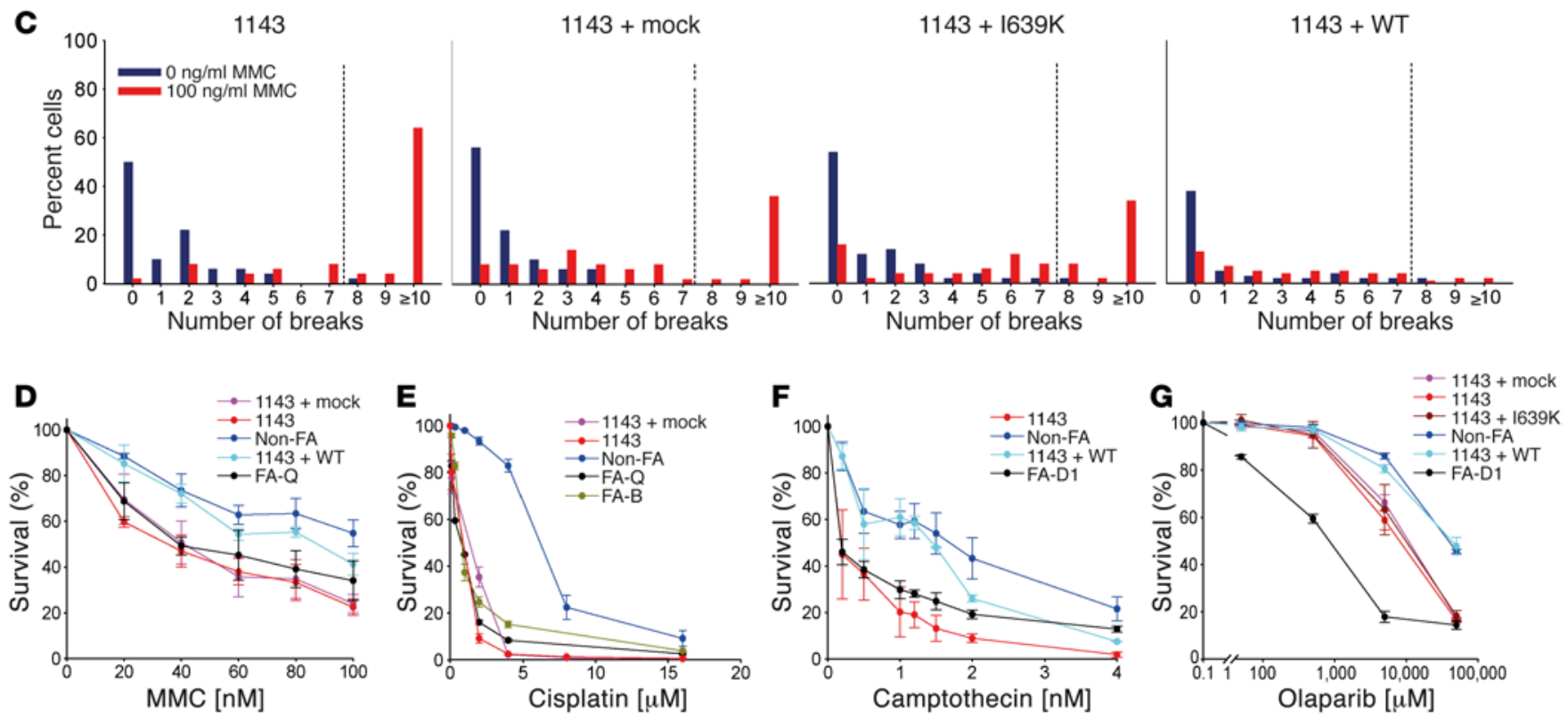

Figure 1. Identification and characterization of RFWD3 mutation. (A) WES data reveal 2 heterozygous sequence variants in the RFWD3 gene, highlighted in green. The gene is located on the minus strand; chromosomal and amino acid positions next to the mutations are depicted atop the sequence data. (B) Sanger sequencing confirms both mutations in individual 1143 and familial segregation. (C) Histograms reflecting proportions of cells with the indicated number of chromosomal breaks per metaphase, blue without, red after exposure to mitomycin C (MMC). Dashed lines delimit high rates (>7) of RFWD3mutated (1143) and unproductively transduced fibroblasts (1143+mock, 1143+l630K). Rates of 1143+WT are rescued. Fifty metaphases each were scored. The 1143 plot is reused in Supplemental Figure 1B. (D-C) Dose-response curves of the same cell lines used in C compared with non-FA and FA control (FA-Q, FA-B, or FA-D1) fibroblasts after exposure to the indicated agents and concentrations. The data represent mean $\pm \mathrm{SEM} ; N=5$. The 1143 and non-FA curves of $\mathbf{D}$ are reused in Figure 2E. The 1143 and non-FA curves of $\mathbf{F}$ are reused in Supplemental Figure 2G.

mock or I639K-mutant vector were sensitive to the poly-ADP ribose polymerase 1/2 (PARP1/2) inhibitor olaparib. This sensitivity was distinct but not as pronounced as that of FA-D1 cells. Once again, this sensitivity was rescued by ectopic expression of WT-RFWD3 (Figure 1G). Poly-ADP ribose mediates the BRCA2dependent early DNA damage response (22). Since RFWD3 deficiency impairs that mechanism, as indicated by cellular olaparib sensitivity, the latter results suggest the involvement of RFWD3 in HR in a BRCA2-dependent manner.

Consequences of RFWD3 deprivation. RFWD3 deficiency provides a means to infer normal protein functions from malfunction. The I-SceI-induced HR assay has become widely accepted as an indicator for a protein to influence the HR mechanism $(23,24)$. In this assay, inactivation by RNA interference of either BRCA2 or 

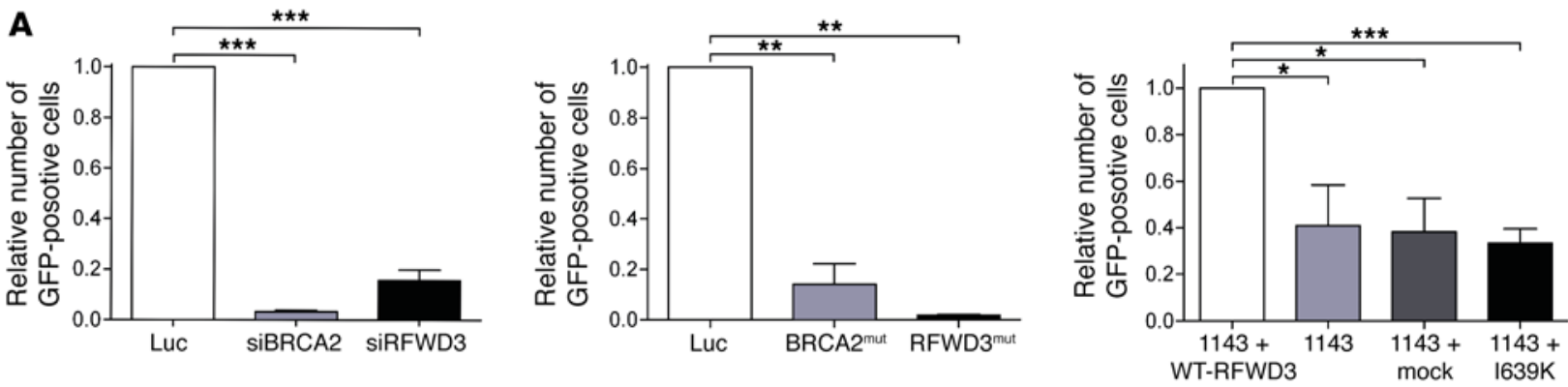

B

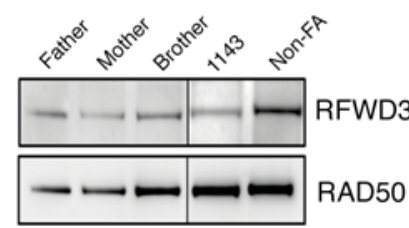

C

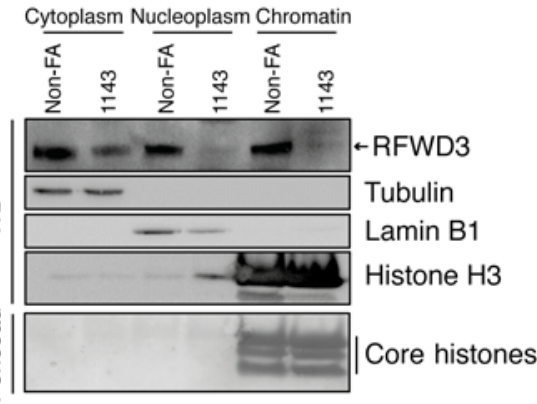

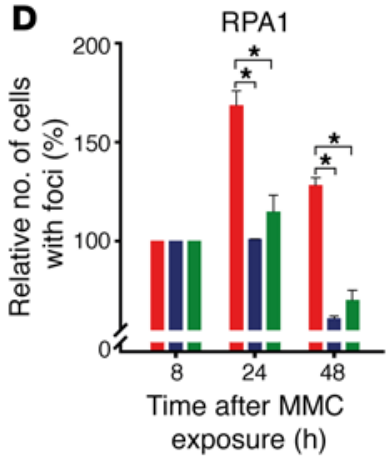
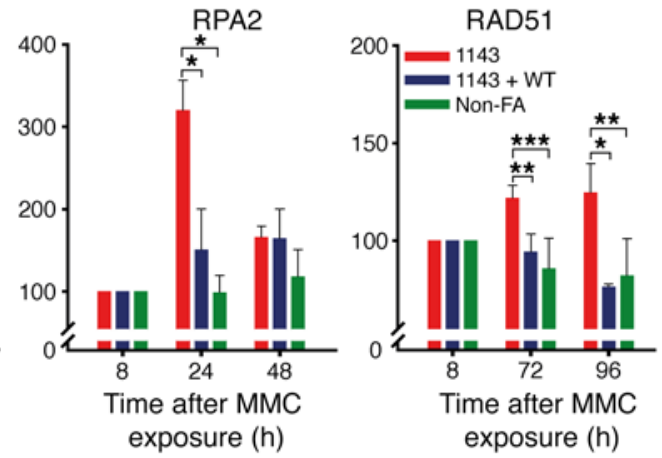
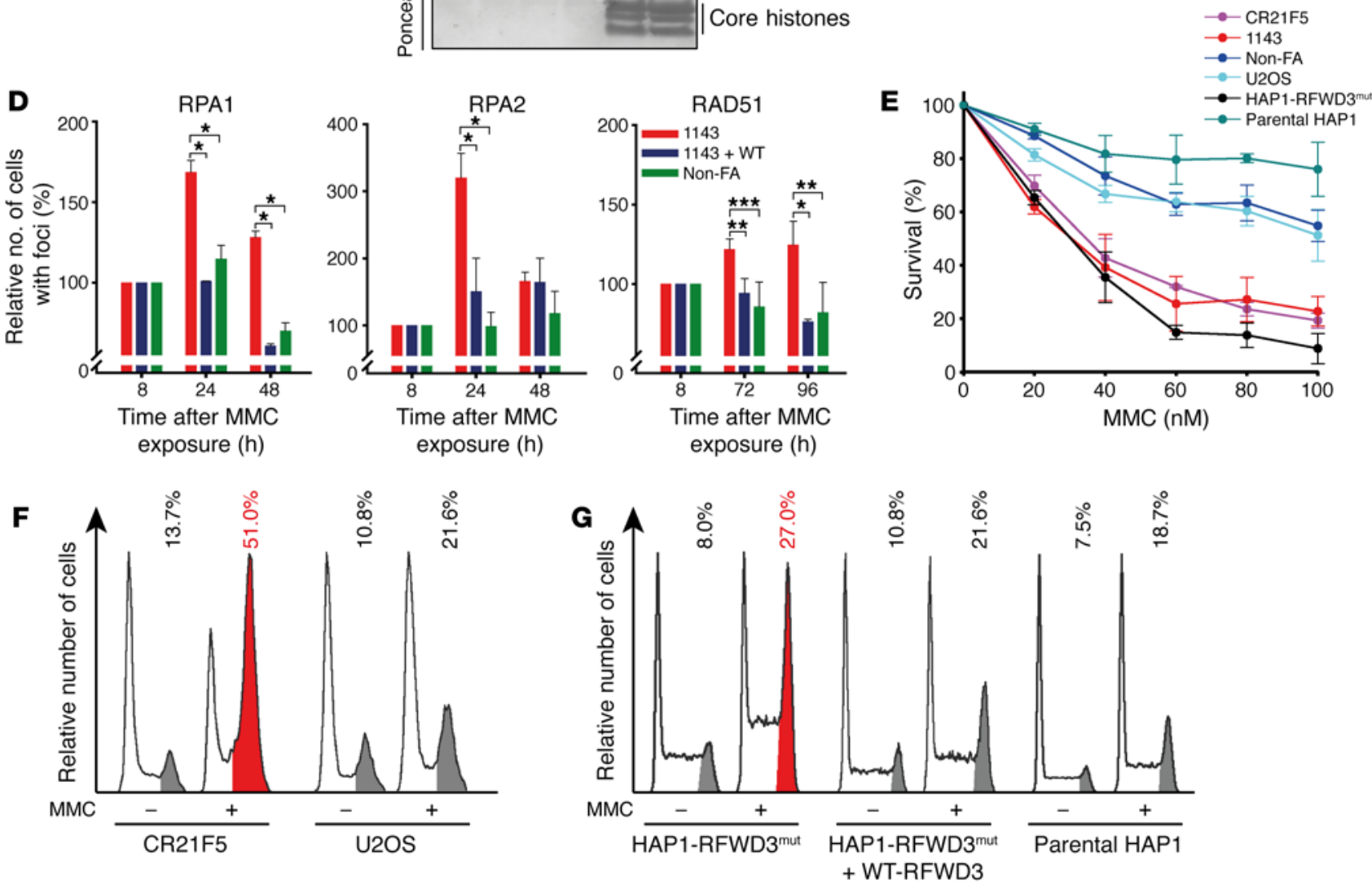

Figure 2. Involvement of RFWD3 in HR and ICL repair. (A) Reduced HR in RFWD3-deficient human cells as signaled by the I-Scel-induced HR assay. Shown is the decrease of GFP-positive (HR-active) cells compared with controls. The left graph shows data from siRNA-transfected U2OS cells (luciferase [Luc; mock] vs. BRCA2 and RFWD3). The middle graph represents data from non-FA versus RFWD3-mutant 1143 and BRCA2/FANCD1-mutant fibroblasts, a disease-control FA-D1 line with the homozygous mutation c.469A>T (p.Lys157*). The right graph displays data from 1143 transduced with WT-RFWD3 versus mock, I639K, and nontransduced 1143 fibroblasts. All results are corrected for transfection rate and size of S phase. (B) Western blot with RFWD3 antibody including lysates from 1143 , her family members, and non-FA fibroblasts exposed to MMC. Lanes were run on the same gel but were noncontiguous. (C) Cell fractionation of protein lysates from non-FA and 1143 fibroblasts exposed to MMC. (D) Proportion of RPA1, RPA2, and RAD51 foci-positive cells in 1143, 1143+WT-RFWD3-transduced, and non-FA fibroblasts at different intervals after an initial 8-hour pulse of MMC exposure. (E) Dose-response curves of CRISPR clone CR21F5 versus parental U2OS cells, HAP1-RFWD3- versus HAP1 control cells, and 1143 versus non-FA fibroblasts exposed to MMC. The 1143 and non-FA curves are reused in Figure 1D. ( $F$ and $\mathbf{G}$ ) Cell cycle analysis regarding $G_{2}$-phase arrest of CRISPR clone CR21F5 versus parental U2OS cells and of HAP1-RFWD3- versus HAP1-RFWD3- ${ }^{-}$WT-complemented and HAP1 control cells without or with exposure to MMC. Increased $G_{2}$ compartment size is highlighted in red; normal size is shown in gray. Data in $\mathbf{A}, \mathbf{D}$, and $\mathbf{E}$ represent mean $\pm \mathrm{SEM} ; N=3$ for siRNA experiments, otherwise $N=5$. ${ }^{*} P<0.05$; ${ }^{* *} P<0.01 ;{ }^{* *} P<0.001$ by unpaired, 2-tailed Student's $t$ test. 
RFWD3 resulted in a highly significant loss of HR function relative to mock siLuciferase (siLuc) transfection, confirming that RFWD3 is involved in the HR pathway (Figure 2A, left panel). Similarly, HR activity of FA-D1 (biallelic BRCA2/FANCD1-mutant) fibroblasts and of 1143 cells was compared with that of human normal control fibroblasts. RFWD3 deficiency downregulated HR activity substantially, resembling BRCA2 deficiency (Figure 2A, middle panel). Untreated, mock-transduced, or RFWD3-I639K cDNAtransduced 1134 fibroblasts were impaired in HR in contrast to 1143 fibroblasts after WT-RFWD3 transduction, confirming in an isogenic system that the RFWD3-I639K mutation of 1143 cells is responsible for the loss of HR activity (Figure 2A, right panel).

Further experiments aimed at understanding in which way the 1143 mutations exert their effects. Western blot analysis of 1143 lymphoblasts showed that mutant RFWD3 of normal size was expressed whereas any truncated protein was undetectable (Figure 2B). The full-length mutant protein may be slightly less stable in cytoplasm, as cellular fractionation studies suggested, but is strikingly less relocated to the nucleus and to chromatin (Figure 2C). Paucity of mutant RFWD3-I639K in chromatin, let alone impaired function, resulted in a longer persistence of RPA and RAD51 nuclear foci in 1143 fibroblasts. After an initial pulse of 8 hours of exposure to MMC, the proportion of 1143 cells that scored positive for RPA foci was increased at early times ( 24 hours and 48 hours; Figure 2D, left and middle panels), and the proportion of RAD51 focipositive cells was elevated at later times (72 hours and 96 hours; Figure 2D, right panel). At other times (RPA foci-positive cells after 48 hours and RAD51 foci-positive cells before 72 hours) there was no significant difference of foci-positive 1143 cells compared with $1143+$ WT complemented and normal control fibroblasts. These data extend the idea that RFWD3 normally promotes HR induced by ICLs, and HR is delayed when RFWD3 is mutated.

Three different cellular model systems recapitulate RFWD3 mutation. Using the CRISPR/Cas9 system, we generated an RFWD3mutant clone of U2OS cells, designated CR21F5, owning the homozygous 6-bp deletion c.1941_1947delCGGCAC. The deletion results in the in-frame loss of 2 amino acids, p.R648_H649del, located in the RPA2-binding WD40 domain. CR21F5 cells displayed increased sensitivity to MMC in survival and cell cycle studies (Figure 2, E and F) and to camptothecin (Supplemental Figure $1 G)$, mimicking 1143 fibroblasts, but contrasting with parental U2OS cells and 1143 WT-RFWD3-complemented fibroblasts.

A second $R F W D 3$-deficient cellular model represents HAP1 cells harboring a 13-bp deletion in RFWD3 exon 3, c.566_578del, predicted to result in the largely mutated and truncated protein RFWD3 p.P189Lfs ${ }^{*} 174$. Consistently, these cells also displayed MMC sensitivity in survival and cell cycle studies (Figure 2, E and G). Unfortunately, HAP1 cells tend to duplicate chromosomes, which limits their use for chromosome breakage studies. RFWD3mutated HAP1 cells were complemented by transduction with WT-RFWD3, demonstrated by rescue from MMC sensitivity in terms of $\mathrm{G}_{2}$ arrest (Figure $2 \mathrm{G}$ ).

Third, we generated $\triangle$ RFWD3 DT40 cells by targeted disruption of the chicken RFWD3 locus (Supplemental Figure 2, A and $B)$. The cells were deduced to be functionally null for 2 reasons: the $\mathrm{CxxC}$ motif in the RING finger was deleted, and no RFWD3 transcript was detected by real-time PCR (Supplemental Figure
2C). $\triangle$ RFWD3 DT40 cells grew more slowly in culture than nontargeted DT40 cells (Supplemental Figure 2D). The gene targeting frequency at the ovalbumin and KU7O loci in $\triangle$ RFWD3 DT40 cells was determined by disruption constructs as previously described (25). Targeting events were greatly reduced in 2 independently produced $\triangle$ RFWD3 versus parental DT40 cell lines (Figure $3 \mathrm{~A}$ ).

Defective HR in $\triangle$ RFWD3 DT40 cells was further examined by a modified I-SceI-induced HR assay. The recombination substrate SCneo (2 tandem nonfunctional neo cassettes that can be repaired by HR) was integrated at the ovalbumin locus. In DT40 cells, transient transfection of I-SceI expression vector resulted in an increase of more than 3 orders of magnitude of neo-resistant cells compared with cells transfected with control vector (Figure 3B). $\triangle$ RFWD3 DT40 cells achieved HR events with approximately 1 order of magnitude less efficiency, which was not improved when they were transfected with chRFWD3-C267A. This mutation disrupts the RING domain, inactivating the RFWD3 E3 ligase function as has been described for the human equivalent mutation C315A in U2OS cells (18). Expression of WT-chRFWD3 rescued the frequency to some extent, while transfection with chRFWD3-I615K, the chicken counterpart of the human I639K mutation, did not. We also determined the ratios of short- and long-tract gene conversion (STGC and LTGC) events (Supplemental Figure 2E) in the HR repair product of SCneo by isolating neo-resistant colonies following I-SceI transduction. STGC and LTGC occurred at indistinguishable rates in nontargeted and $\triangle$ RFWD3 DT40 cells, suggesting that loss of RFWD3 affects efficiency, but not the tract length, of HR induced by DNA double-strand breaks (Supplemental Figure 2F).

$\triangle$ RFWD3 DT40 cells proved hypersensitive to MMC or cisplatin in terms of survival, compared with parental DT40 cells (Figure 3, C and D). Resistance of $\triangle$ RFWD3 DT40 cells to these agents was rescued by stable expression of WT-chRFWD3, but not of chRFWD3-C267A. Transfection with chRFWD3-I615K resulted in intermediate sensitivity consistent with this mutation being hypomorphic in humans. Chromosomal studies revealed increased breakage in the form of chromatid-and chromosome-type lesions in $\triangle$ RFWD3 DT40 compared with parental DT40 cells when exposed to MMC (Figure $3 \mathrm{E}$ ), resembling $\triangle \mathrm{FANCD} 2$ or $\triangle \mathrm{XRCC} 3 \mathrm{DT} 40$ cells (26). Again, stable expression of WT-chRFWD3 restored the breakage rate to normal, whereas transfection with chRFWD3-C267A had virtually no effect and expression of chRFWD3-I615K resulted in intermediate rates. These results show that cells with the chI615K (equivalent to human I639K) mutant are impaired in HR, in the maintenance of challenged chromosomal integrity, and in MMC and cisplatin tolerance.

Collectively, these 3 different cellular models, all lacking RFWD3 on a different genetic background, support the notion that $R F W D 3$ functions in the FA pathway and in HR.

RFWD3-I639 is necessary for RFWD3 focus formation. In order to examine how the chicken I615K mutation (equivalent to human I639K) affects functions of the RFWD3 protein in cells, $\triangle$ RFWD3 DT40 cells were transiently transfected with mock vector or vector containing GFP-tagged chRFWD3-C267A or the double mutant GFP-chRFWD3-C267A/I615K. The expression of GFPchRFWD3-C267A showed RFWD3 protein that was relocated to the nucleus and induced RFWD3 focus formation (Figure $3 \mathrm{~F}$ ). By contrast, expression of the GFP-chRFWD3-C267A/I615K double 

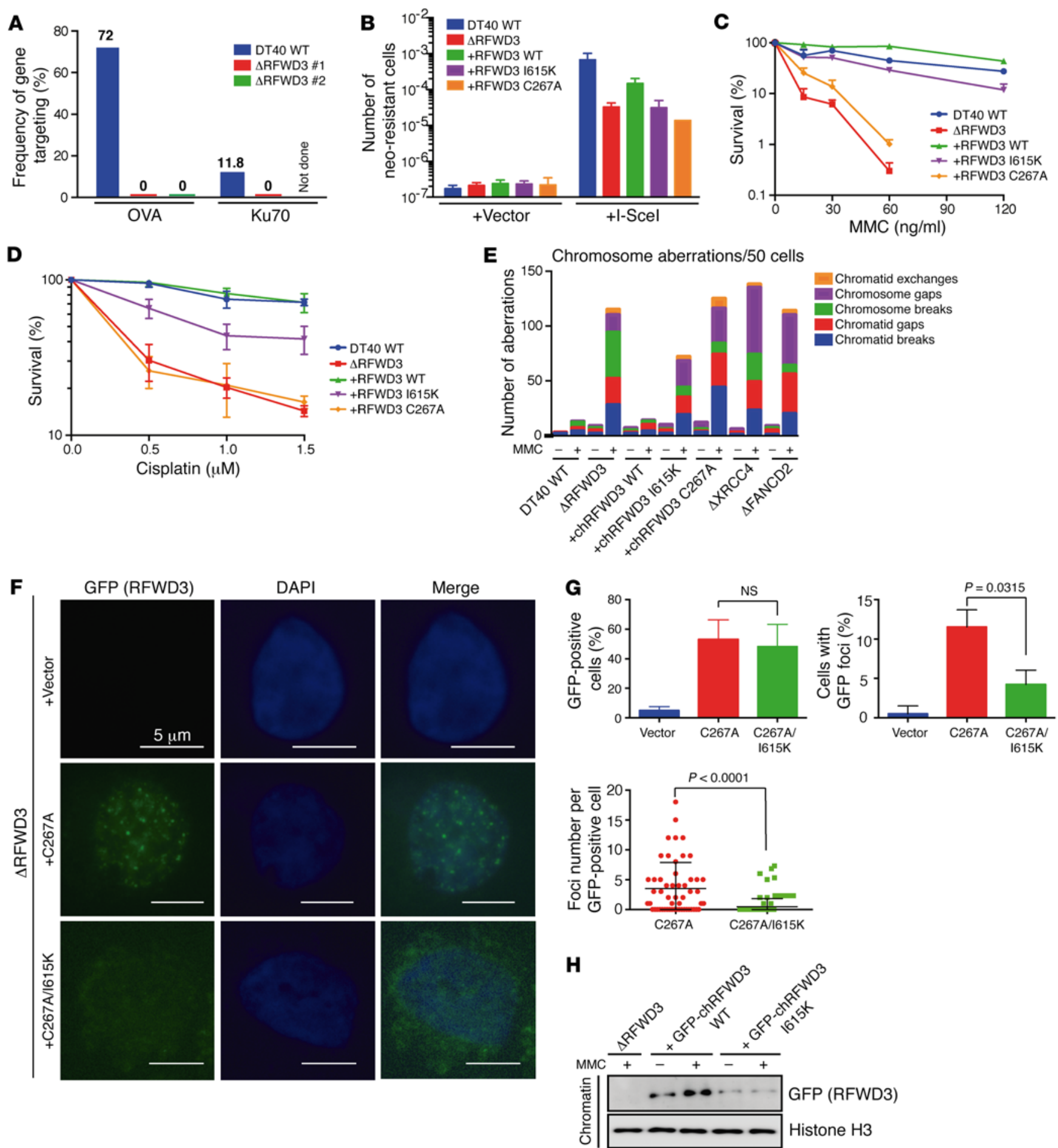

Figure 3. Functional analyses of $\triangle$ RFWD3 DT40 cells. (A) Frequency of gene targeting at the ovalbumin (OVA) and KU70 loci of WT DT40 cells (blue) and 2 independently generated $\triangle$ RFWD3 DT40 cell lines (\#1, red; \#2, green). Percentages of the targeting events relative to the number of examined clones are shown on top of each bar. (B) Frequency of neo-resistant DT40 colonies due to HR events in the SCneo recombination substrate integrated in the OVA locus. Cells with indicated genotypes were transiently transfected with empty vector (+Vector) or with vector containing I-Scel (+I-Scel) and selected in medium containing G418. After 10-14 days the number of colonies was counted. (C and $\mathbf{D})$ Dose-response curves of WT DT40 cells versus $\triangle R F W D 3$ DT40 cells and $\triangle R F W D 3$ DT40 cells transfected with WT-RFWD3, RFWD3-I615K, or RFWD3-C267A. Cells were exposed to MMC or cisplatin. (E) Histograms reflecting proportions of cells with the indicated number of chromosomal lesions per metaphase in WT DT40 and mutant cells with the indicated genotypes and transfections without or with exposure to MMC. Fifty cells each were scored. (F) GFP immunofluorescence analysis of $\triangle R F W D 3$ DT40 fibroblasts transiently transfected with mock, GFP-chRFWD3C267A, or the double mutant GFP-chRFWD3-C267A/I615K after exposure to MMC. (C) Proportion of GFP-RFWD3-positive $\triangle$ RFWD3 DT40 cells (top panel) and GFP-RFWD3 foci-positive $\triangle$ RFWD3 DT40 cells ( $>5$, middle panel) and number of GFP-RFWD3 foci in individual GFP-RFWD3 foci-positive $\triangle$ RFWD3 DT40 cells (bottom panel) after transfection with the indicated plasmids. Cells are those used in F. (H) Relocation of GFP-RFWD3 in $\triangle$ RFWD3 DT40 cells transfected with the indicated plasmids without and with exposure to MMC. Fractions were probed using anti-GFP and anti-histone 3 antibodies. Data in B-D and G represent mean \pm SEM; $N=3$. Significance in G determined by unpaired, 2-tailed Student's $t$ test. NS, not significant. 
mutant prevented the translocation of RFWD3 to the nucleus and focus formation. In this experiment, the percentage of GFPpositive cells was comparable between cells expressing GFPchRFWD3-C267A and GFP-chRFWD3-C267A/I615K (Figure 3G, top panel), indicating that expression levels were similar. However, GFP foci-positive cells were much decreased in the C267A/ I615K double mutant relative to the C267A mutant (Figure 3G, middle panel). Even more strongly reduced was the number of foci in individual GFP-RFWD3 foci-positive cells (Figure 3G, bottom panel). Immunoblotting confirmed that GFP-WT-chRFWD3 is able to access chromatin whereas the level of GFP-chRFWD3I615K in chromatin was much less (Figure $3 \mathrm{H}$ ), paralleling our findings in human RFWD3-mutant 1134 cells.

These results in DT40 cells were supported and extended by studies of human cells. Reduced amounts of FLAG-RFWD3-I639K in chromatin compared with FLAG-WT-RFWD3 were observed on immunoblots when U2OS cells were transiently transfected with corresponding vectors and exposed to MMC (Figure 4A). This notion was underscored by the reduction of RFWD3 focipositive cells, but not RPA2 foci-positive cells, when the $1639 \mathrm{~K}$ mutant was transfected (Figure 4B). Quantification confirmed that there was a highly significant difference in the number of FLAG-RFWD3 foci in individual FLAG-RFWD3 foci-positive cells (Figure 4C, top panel), but there was no difference in the proportion of RPA foci-positive U2OS cells after exposure to MMC (Figure $4 \mathrm{C}$, bottom panel). Coimmunoprecipitation of human FLAGRFWD3-I639K with RPA2 displayed a largely decreased physical interaction in U2OS cells after exposure to MMC, which was in contrast to the transfection with FLAG-WT-RFWD3 (Figure 4D). Pull-down studies between GFP-RFWD3 containing the RING mutation C315A, or the RING mutation plus the patient's WD40 mutation (I639K), and human his-RFWD3-C315A showed that neither mutation affects RFWD3-RFWD3 dimerization (Figure $4 \mathrm{E})$. However, the RING mutation C315A abrogated RFWD3 autoubiquitination whereas the WD40 mutation I639K did not when U2OS cells were transiently transfected with FLAG-RFWD3-WT, FLAG-RFWD3-I639K, or FLAG-RFWD3-C315A and exposed to the proteasome inhibitor MG132 (Figure 4F).

Thus we conclude that RFWD3-I639 or its chicken equivalent chRFWD3-I615 is an absolute requirement for chromatin translocation, physical interaction with RPA, and ICL repair/HR functions of RFWD3.

In U2OS cells FLAG-RFWD3 and FANCD2 foci occur in overlapping or immediate adjacent positions in a majority of cells after exposure to MMC, suggesting temporary cooperation of the proteins in HR (Figure $4 \mathrm{G}$ and Supplemental Figure 2G). This is similar to observations that, following DNA damage induction, a majority of GFP-FANCD2 and RAD51 foci colocalized although RAD51 and FANCD2 foci formation are independent events (27), and that monoubiquitinated FANCD2 and BRCA2/FANCD1 colocalize in DNA damage-inducible foci (28). Together these data indicate that FANCD2 and RFWD3, RAD51, and BRCA2/FANCD1 converge and accumulate at the same DNA damage sites.

We depleted FANCD2 in HAP1 RFWD3-mutant cells, confirmed this by immunoblotting (Supplemental Figure 2, H and I), and exposed siLuc- and siFANCD2-treated RFWD3-mutant HAP1 cells to MMC. Although the $\triangle R F W D 3-H A P 1+s i D 2$ dose-response curve runs below the $\triangle R F W D 3-H A P 1+$ siLuc curve for all concentrations assayed, the differences are small (Figure $4 \mathrm{H}$ ). FANCD2 knockdown appears to have a small additive effect to RFWD3 deficiency, and FANCD2 and RFWD3 may be partially nonepistatic in terms of ICL repair, but a potential nonepistatic effect is borderline and significant only at $10 \mathrm{ng} / \mathrm{ml}$ MMC. A similar observation has been made in DT40 cells that were double-mutant for FANCC whole gene deletion and BRCA2 C-terminal deletion. Such Fancc/ Brca2 $\triangle C T D$ double-mutant cells showed a more severe level of sensitivity to cisplatin and MMC compared with either of the single mutants (27). The additive sensitivity indicates that FANCD2 and RFWD3, or FANCC and BRCA2, may have some nonoverlapping functions in ICL repair.

An Rfwd $3^{-/}$mouse model resembles other FA mouse models. $R f i v d 3$ conditional knockout mice were generated by flanking of exon 3 with loxP sites via gene targeting in Bruce 4 C57BL/6 embryonic stem (ES) cells (29). Gene-targeted ES cell clones were identified and cells then injected into goGermline blastocysts (30). Male goGermline mice were bred to C57BL/6 females to establish heterozygous germline offspring on C57BL/6 background. Cremediated deletion of exon 3 introduced a translational frameshift (Supplemental Figure 3A). Southern blot analysis was used to genotype the offspring (Supplemental Figure 3B). Real-time PCR analysis of normalized relative $R$ fiwd 3 mRNA expression in $R f w d 3^{-/}$and $R f w d 3^{+/+}$mice confirmed marked differences. Virtually no amplification of an $R$ fwd 3 mRNA region bridging exons 3-5 was observed in $R f w d 3^{-/-}$mice compared with $R$ fwd $3^{+/+}$controls (Supplemental Figure 3C). This confirmed the deletion of exon 3 at the mRNA level. Further results demonstrated that some Rfivd 3 mRNA survives despite the removal of exon 3. Transcript levels upon amplification of an Rfwd3 mRNA region bridging exons 6-8 were approximately 10-fold less in $R f w d 3^{-/-}$compared with $R{f i w d 3^{+/+}}$samples (Supplemental Figure 3D). However, the loss of exon 3 set downstream exons out of the Rfivd3 reading frame and rendered them nonfunctional. An initial characterization of $R f w d 3^{--}$mice demonstrated that they are viable and do not show overt phenotypic abnormalities. Heterozygous and homozygous offspring were born at approximately 1:1 sex ratio (Figure 5A). $R \mathrm{fwd}^{+/-}$heterozygous mating revealed a deficit of $R \mathrm{fw}^{\mathrm{w}} \mathrm{3}^{-/-}$pups (18.7\% observed vs. $25 \%$ expected). This tendency may be due to embryonic lethality, but the difference was not statistically significant because of small mouse numbers. Subfertility was an additional finding. Three types of test mating including $R f i w d 3^{-/-}$ mice were attempted. Attempts were made to mate $3 \mathrm{KO}$ males to $3 \mathrm{KO}$ females, $3 \mathrm{WT}$ males to $3 \mathrm{KO}$ females, and $3 \mathrm{KO}$ males to 3 WT females. Ample time allowed, none of these efforts produced pups, a rate that is incompatible with spontaneous mating failure among $R$ fwd $^{+/+}$or $R \mathrm{fwd}^{+/-} \mathrm{C} 57 \mathrm{BL} / 6$ mice. By these studies, $6 \mathrm{KO}$ females $(23.1 \%$ of a total of $26 \mathrm{KO}$ mice) and $3 \mathrm{KO}$ males $(11.5 \%$ of a total of $26 \mathrm{KO}$ mice) proved infertile, as mating of $3 \mathrm{KO}$ males to both $\mathrm{KO}$ and WT females was attempted. Although more, if not all, $R \mathrm{fwd}^{-/-}$mice may be infertile, we refer to our observation for now as subfertility, since some $R$ fw $d 3^{-/-}$mice were not mated but were used for other studies. Histological examination of $R f_{w d 3^{-1-}}$ mice detected testicular and ovarian atrophy. In males, seminiferous tubules were largely retained. There was a patchy change where a few showed only small amounts of spermatogenesis, but 
A

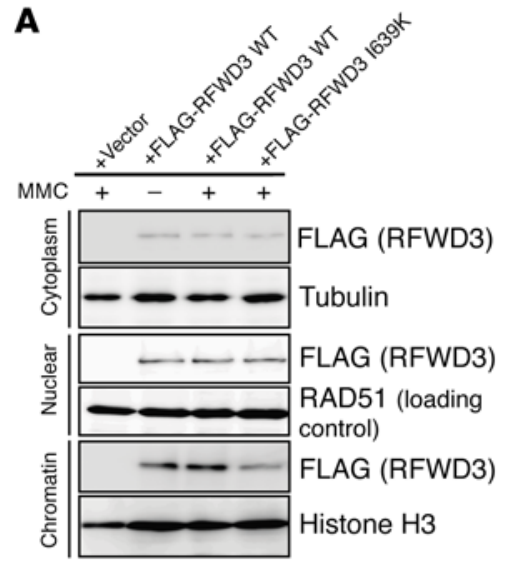

C
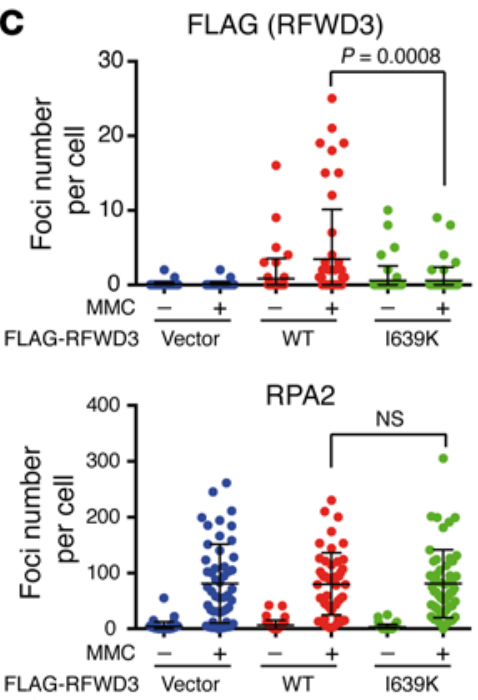

$\mathbf{F}$

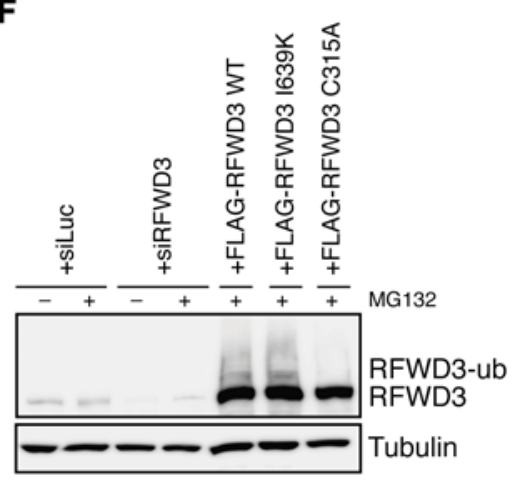

B
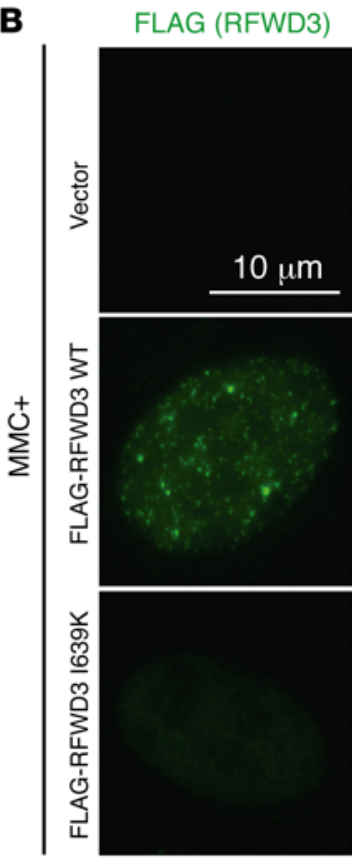

RPA2

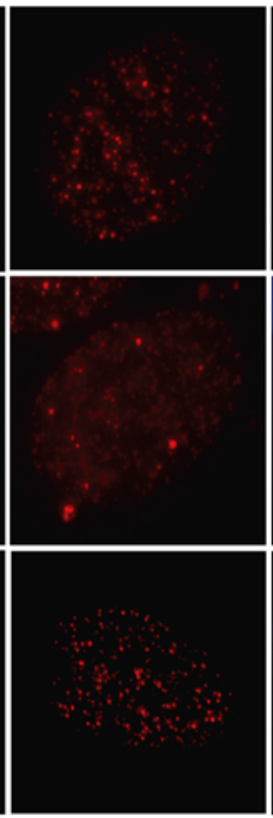

Merge (+DAPI)

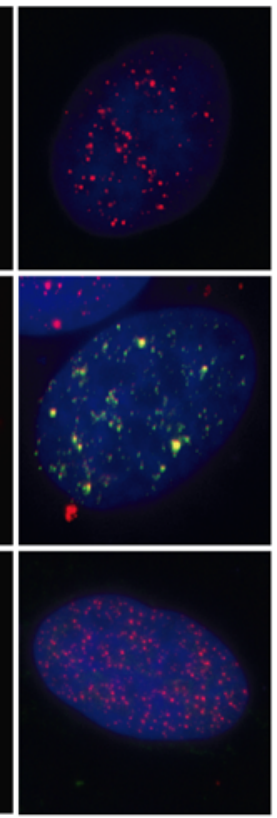

D

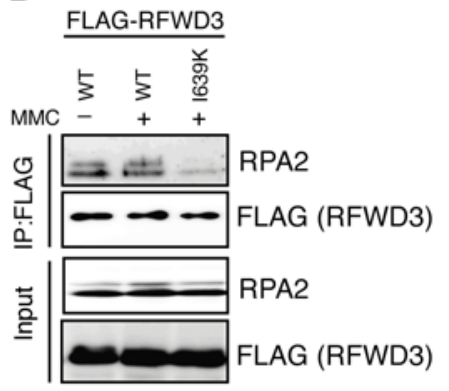

E GFP-RFWD3

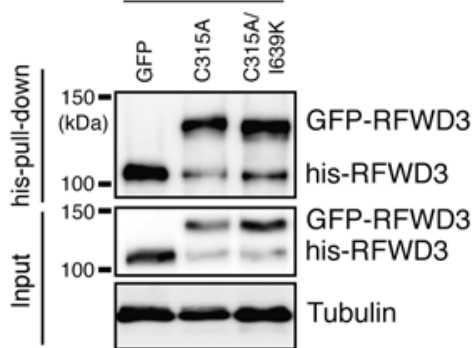

G FLAG (RFWD3)

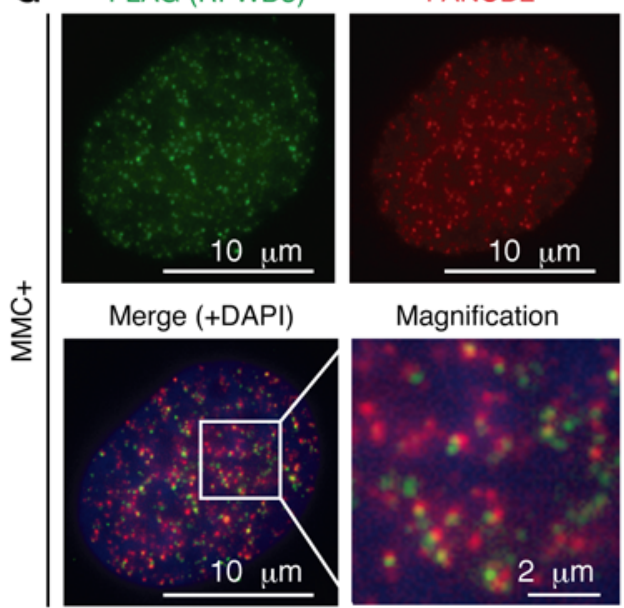

H

- WT + siLuc

$-W T+\operatorname{siD} 2$

$-\triangle R F W D 3+$ siluc

$-\triangle R F W D 3+\operatorname{siD} 2$

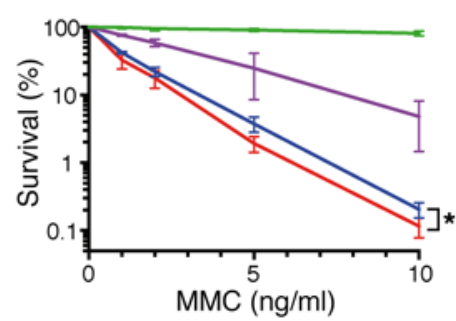


Figure 4. Interaction studies of human RFWD3. (A) Cell fractionation shows impaired chromatin relocation of RFWD3 with the mutation in the WD40 domain when U20S cells were transiently transfected with FLAGWT-RFWD3 or FLAG-RFWD3-I639K and exposed to MMC as indicated. (B) FLAG-RFWD3 immunofluorescence analysis of the same cells used in $\mathbf{A}$ exposed to MMC. Anti-FLAG detected RFWD3, anti-RPA2 detected endogenous RPA, and DAPI counterstains the nucleus. (C) Number of FLAG-RFWD3 (top graph) and RPA2 (bottom graph) foci per nucleus without or with exposure to MMC. Cells are those used in A. More than 50 cells were counted for each sample. The experiment was repeated 4 times. Data represent mean \pm SD. $P$ values of WT versus $1639 \mathrm{~K}$ were calculated using the unpaired, 2-tailed Student's $t$ test. NS, not significant. (D) Coimmunoprecipitation of FLAG-WT-RFWD3 or FLAG-RFWD3-I639K with RPA2 in transiently transfected U2OS cells without or with exposure to MMC. (E) Pull-down study of GFP-RFWD3 and his-RFWD3 dimerization in U2OS cells transiently transfected with GFP, GFP-RFWD3-C315A (RING mutation), or GFP-RFWD3-C315A/I639K (double mutation). Detection was by anti-his and anti-GFP antibodies. (F) Auto-ubiquitination of FLAG-WTRFWD3, FLAG-RFWD3-I639K, or FLAG-RFWD3-C315A in transiently transfected U2OS cells without or with exposure to the proteasome inhibitor MG132. siLuc and siRFWD3 served as negative controls. Detection was by anti-RFWD3. (G) Colocalization of FLAG-RFWD3 and FANCD2. U2OS cells were transiently transfected with FLAG-RFWD3 and exposed to MMC (100 $\mathrm{ng} / \mathrm{ml}$ for 24 hours). (H) Survival curves of WT HAP1 and RFWD3-mutated HAP1 cells transfected with siRNAs to luciferase (Luc) or FANCD2 (D2) and exposed to MMC. Means $\pm S D$ of 3 independent experiments are shown. Depletion of FANCD2 and BRCA2 (control) was analyzed by immunoblotting and is shown in Supplemental Figure $2, \mathrm{H}$ and $\mathrm{I}$. The $P$ value $\left({ }^{*} P<\right.$ $0.05)$ of $\triangle R F W D 3+$ siLuc versus $\triangle R F W D 3+\operatorname{siD} 2$ was calculated using the Tukey's range test.

most appeared empty with little evidence of any active cell divisions (Figure 5B). Female $R$ fwd $^{-/-}$mice displayed small ovaries with predominance of luteal cells and inactive epithelium on the surface. No follicular differentiation or development was detected (Figure 5C). As a preliminary observation, some $R$ fw $\mathrm{d}^{-/-}$offspring lived a shortened lifespan for yet unknown reasons.

Rfw $d 3^{-/-}$mouse embryonic fibroblasts (MEFs) proved hypersensitive to DNA cross-linking agents. When exposed to MMC they showed increased $\mathrm{G}_{2}$-phase accumulation compared with $R \mathrm{fwd}^{+/+}$MEFs (Figure 5D). Chromosomal breakage analysis of $R \mathrm{fwd}^{-/-}$MEFs revealed increased numbers of lesions with a distribution typical for FA, in contrast to $R f w d 3^{+/+}$mice, which showed unremarkable rates and distribution (Figure $5 \mathrm{E}$ ). Breaks were mostly of the chromatid type and included characteristic radial figures (Figure $5 \mathrm{~F}$ ). $R$ fiwd $3^{-/-}$MEFs also showed reduced dose-dependent survival when exposed to MMC compared with $R f w d 3^{+/+}$MEFs, and they were rescued by prior transduction with human WT-RFWD3 (Figure 5G).

\section{Discussion}

This study presents a new FA subtype resulting from biallelic mutations in the E3 ubiquitin ligase gene RFWD3. Two compound heterozygous mutations have been identified using WES. The paternally derived allele shows a duplication of the cytosine residues at positions c.205_206 leading to premature termination of translation. Because we did not detect truncated RFWD3 protein and the mutation is $\mathrm{N}$-terminally located, it is safe to assume that the transcript is subject to nonsense-mediated RNA decay. The maternal allele produced a relatively stable RFWD3 protein with the missense substitution p.I639K. This is located in the pre- dicted WD40 domain (18). We showed it to be pathogenic in different systems, as it prevents RFWD3 relocation to chromatin and impairs several of its functions.

Elia et al. (14) recently reported, in a context independent of FA, that RFWD3 ubiquitinates RPA. Our work shows disrupted physical interaction of mutant RFWD3 and RPA. This RFWD3RPA malfunction provides a mechanistic insight primarily from an observation in humans and brings another missing piece of the jigsaw regarding the pathogenesis of FA to fall into place. Only one side of the coin is that the RFWD3-I639K mutation abrogates the E3 ligase function toward RPA2, since the RPA2 interaction domain is located at the C-terminus of the RFWD3 protein (31) that includes the I639 residue. Further studies will elucidate the exact mechanisms. Moreover, it remains to be seen whether other interactors or processes are implicated. Since RPA is involved in HR, our studies were aimed at the demonstration that this holds also for RFWD3. Two experimental results supported this view. The results of the I-SceI-induced HR assay in human and chicken cells globally indicated impaired homologydirected recombination upon RFWD3 mutation. Prolonged retention of RPA1 and RPA2 foci in cells with the I639K substitution suggested delayed HR. The finding that RAD51 foci also persisted longer as in controls may indicate an indirect effect on, or a direct implication of, RAD51.

$\mathrm{HR}$ is a hierarchical process with master players such as the BRCA1-PALB2-BRCA2 complex and RAD51, and it is also a linear pathway that proceeds sequentially step by step. Impaired HR can result in an FA phenotype as has been demonstrated by mutations of genes contributing to HR that are also FA genes (32). Including $R F W D 3$, the number of reported FA genes involved in HR amounts to a remarkable total of 7 , which act at different stages of HR. While the BRCA1, PALB2, and BRCA2 proteins function at the level of orchestration, RFWD3 works at middle-phase HR after single-stranded DNA marking, before late-HR factors come into play including RAD51C and XRCC2 that have been associated with strand invasion, D-loop formation, and Holliday junction resolution (33). This leads us to conclude that the FA/BRCA pathway converges on HR. Notably, it is not the frequently mutated FA genes whose products are among those that form the FA core complex that have most contributed to recognition of the association of FA and HR for genomic stability in humans, including cancer prevention and longevity. This has rather been achieved by the more recent observation of inactivation of newly identified downstream or late FA genes with immediate involvement in the excision of ICLs, in translesion synthesis or eminently in HR. These less frequently affected FA genes have all been reported initially with few or single patients and hypomorphic mutations, because other constellations may be nonviable. Nevertheless, this way we have learned more of the FA/BRCA pathway than was discovered over a long period with the upstream or early FA genes.

The proposed patient demonstrates that RFWD3 deficiency does not necessarily result in early tumorigenesis, partly perhaps because the p.I639K mutation is not fully penetrant. On the other hand, 1143 patient cells do clearly show impaired HR activity, which evidently does not drive forth early-onset embryonal tumors or acute myeloid leukemia. Pronounced early cancer proneness has been reported only for FA due to 

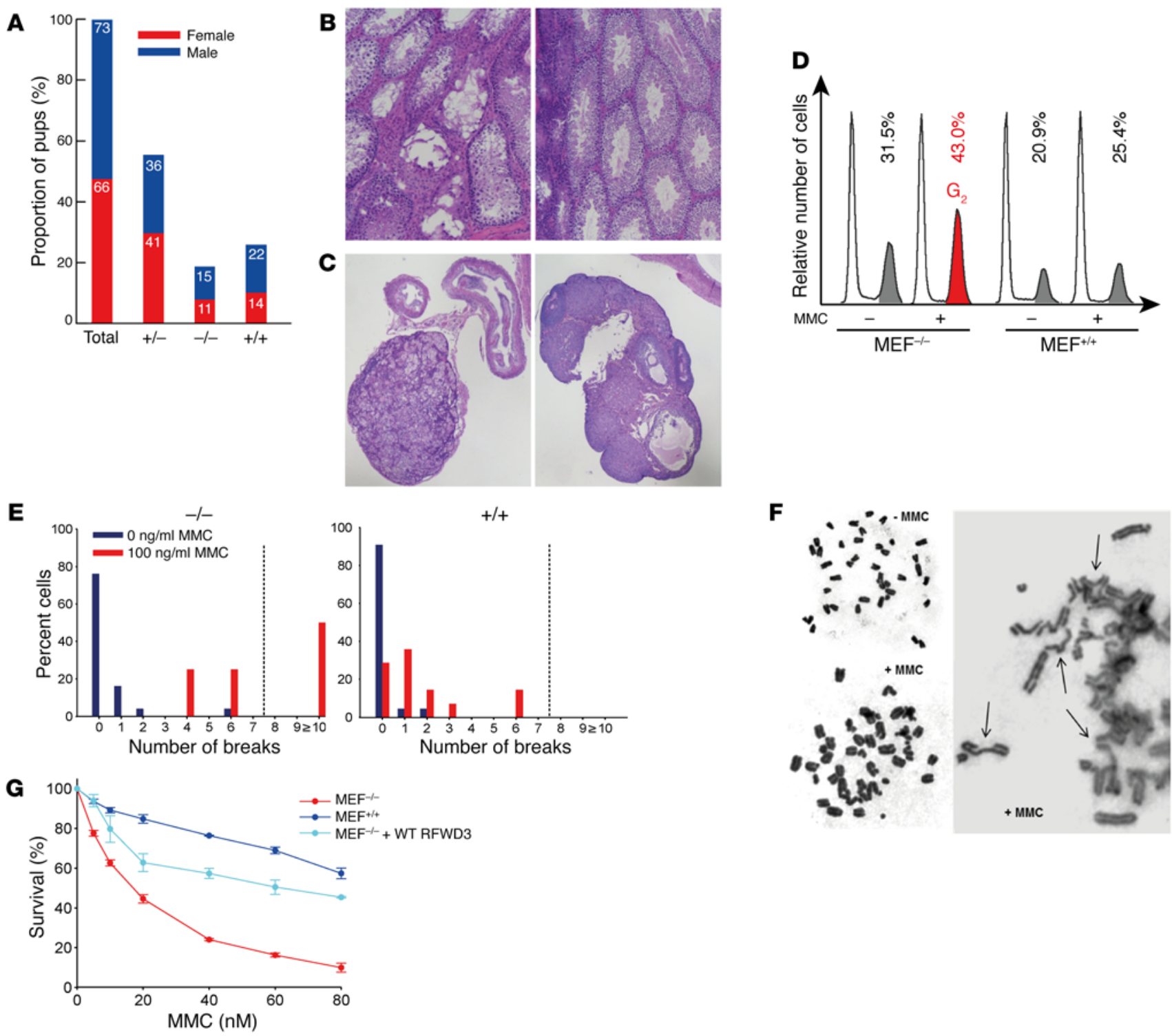

Figure 5. Characterization of an $R f w d 3^{-/-}$mouse model. (A) Numbers and proportions of female (red) and male (blue) $R f w d 3^{+/-}, R f w d 3^{-/-}$, and $R f w d 3^{+/+}$ mouse offspring of heterozygous mating. $R f w d 3^{-/-}$pups were born at a sub-Mendelian rate. (B) Left panel displays atrophic $R f w d 3^{-/-}$mouse testis. Remaining seminiferous tubules have little active spermatogenesis and few spermatozoa. Right panel shows $R f w d 3^{+/+}$testis with normal spermatogenesis for control. The original micrographs were taken at $\times 40$. (C) Left panel displays atrophic $R f w d 3^{-/-}$mouse ovary. There are clusters of luteal cells within a delicate stroma, but there is no follicular development. Right panel shows $R f w d 3^{+/+}$ovary with normal follicular development for control. The original micrographs were taken at $\times 40$. (D) Cell cycle analysis examining $\mathrm{G}_{2}$-phase arrest in $R f w d 3^{-/-}$versus $R f w d 3^{+/+}$MEFs without or with exposure to MMC. Increased $\mathrm{G}_{2}$ compartment size is highlighted in red, normal size shown in gray. (E) Histograms reflecting proportions of cells with the indicated number of chromosomal breaks per metaphase, blue without, red after exposure to MMC, in $R f w d 3^{-/-}$and $R f w d 3^{+/+}$MEFs. Fifty cells each were scored. Sensitivity is indicated by the high rate of metaphases with more than 7 breaks (dashed line). Fifty metaphases each were scored. (F) Micrographs of $R f$ wd $3^{-1-}$ MEF metaphase preparations. Unaffected cell without prior exposure to MMC (top left), increased chromosomal breakage after exposure to MMC (bottom left), and predominance of chromatid-type lesions and radial reunion figures (arrows, right) after exposure to MMC. The original micrographs were taken at $\times 100$. (C) Dose-response curves of $R f w d 3^{-/-} \mathrm{MEFs}, R f w d 3^{+/+}$MEFs, and $R f w d 3^{-/-}$MEFs transduced with human WT-RFWD3 exposed to MMC. The data represent mean $\pm \mathrm{SEM} ; N=3$.

biallelic mutations of BRCA2 (FA-D1) (34) and of the direct BRCA2 interactor PALB2 (FA-N) (35). Our study of RFWD3 deficiency extends observations in patients with FA subtypes FA-O (RAD51C/FANCO-mutant) and FA-U (XRCC2/FANCUmutant), whose cells are deficient in HR, too, but patients have not been described with early embryonal tumors $(32,36,37)$. Moreover, distinct early tumorigenesis has not been associated with FA-R (RAD51/FANCR-mutant) or FA-S (BRCA1/FANCSmutant) patients $(19,20,38)$, whose affected genes/proteins likewise are involved in HR. We suggest that BRCA2-specific rather than FA/BRCA pathway functions facilitate the extreme early proneness to malignancies in FA patients of groups FA-D1 and FA-N, which is much greater than and different from the tumor disposition inherent to other FA subtypes $(1,4)$. 
This is dissimilar to synthetic lethality that affects all cells with deficient HR. Synthetic lethality occurs when there is a potent and lethal synergy between 2 otherwise nonlethal events: in this context, 1 element is camptothecin, a highly specific DNA topoisomerase I inhibitor that acts to maintain single-strand breaks generated by topoisomerases during replication (39), or olaparib, a PARP inhibitor that also blocks repair of DNA single-strand breaks (40). Unrepaired single-strand breaks are converted to doublestrand breaks upon replication. The other component of synthetic lethality in our study is RFWD3 deficiency that leads to loss of HR function necessary to repair lesions such as double-strand breaks correctly. As they cannot be properly repaired, HR-deficient cells prove to be selectively and exceptionally sensitive to compounds that exploit their Achilles' heel, which results in unsalvageable DNA damage and consequent cell lethality. Both topoisomerase I and PARP inhibitors also form protein-DNA complexes that are highly toxic to cells because they block DNA replication. Apart from RFWD3 deficiency, increased sensitivity to this type of chemicals is shared by cells with biallelic mutations of the other 6 FA genes acting on HR.

We generated an $\mathrm{Rfw} \mathrm{3}^{---}$mouse model that serves as direct support of the pathogenicity of mutant RFWD3 in the human disease FA. The $R f w d 3^{-/-}$mouse model may also serve as a future basis for a better understanding of patient-independent in vivo functions of RFWD3. The fact that $R f w d 3^{-/-}$mice fail to show a recognizable pattern of phenotypical anomalies that resemble those in FA patients is compatible with this view, as it is mirrored in other FA KO mouse models (41-43). Nonetheless, subfertility and embryonic lethality are features frequently seen in other FA mouse models (41). No matter whether $R f w d 3^{-/-}$mice are completely sterile or not, it is a trait in which they resemble either recently reported $\mathrm{Fancp}^{-1-}$ mice or other FA mouse models (41-43). Rfwd $3^{-/-}$mice, or their cells, may serve for studies of attrition of hematopoietic stem cells in FA, for transplantation studies, for sensitivity testing of aldehydes and oxygen, and for drug screens. Long-term survival observations are necessary for $R f w d 3^{-/-}$mice to settle a susceptibility to malignancies.

In conclusion, we identified biallelic mutations of RFWD3 in an individual presenting with classical FA. As an E3 ubiquitin ligase that promotes HR via ubiquitination of RPA, RFWD3 finds its position late in the FA/BRCA pathway, downstream of BRCA2/ FANCD1. Since HR is hampered and delayed when RFWD3 is mutated, RFWD3 must be regarded as a critical component probably in the regulation of that complex process. In recognition of its function as an FA gene we propose to assign RFWD3 the alias $F A N C W$. Further clinical studies will reveal whether it is also a susceptibility gene for familial cancer such as hereditary breast and ovarian cancer (HBOC) disease when mutated on only 1 allele, as holds true for many downstream FA genes (44).

\section{Methods}

Cell lines. All cells except DT40 were cultured in complete DMEM supplemented with $15 \% \mathrm{FBS}$ at $37^{\circ} \mathrm{C}$ in incubators with $5 \% \mathrm{CO}_{2}$. Cell immortalization and DT40 maintenance were according to standard protocols. All cell lines used in this study are summarized in Supplemental Table 3.

DNA sample preparation, PCR, and Sanger sequencing. Genomic DNA was isolated using the GeneJet Genomic DNA Purification Kit (Fermentas) or the Quick-gDNA MiniPrep Kit (Zymo Research).
Mutation validation, segregation analysis, and screens for off-target events were performed by Sanger sequencing using Bio-X-Act DNA short polymerase (Bioline) according to the manufacturer's protocol on an Applied Biosystems 3130xl sequencer. Primer sequences for genomic sequencing are listed in Supplemental Table 4. Primer design and mutation nomenclature are based on transcript ENST00000361070.8. Touchdown PCR was performed with lid heating at $98^{\circ} \mathrm{C}$, sample preheating at $95^{\circ} \mathrm{C}$ for 3 minutes, 10 cycles with annealing temperatures from $65^{\circ} \mathrm{C}$ to $55^{\circ} \mathrm{C}$ followed by 25 cycles with denaturation at $95^{\circ} \mathrm{C}$, annealing at $55^{\circ} \mathrm{C}$ and elongation at $72^{\circ} \mathrm{C}$, and a final elongation step at $72^{\circ} \mathrm{C}$ for 5 minutes.

Whole exome sequencing. Target enrichment was performed with the Agilent SureSelect Human All Exon 50Mb Kit and followed by nextgeneration sequencing (NGS) on a HighSeq2000 instrument (Illumina). Processing of WES data using NextGENe software v2.18 (Softgenetics) and in silico analysis were performed as described before (45).

Exome sequencing data. The NGS sequencing data were deposited in the European Genome-Phenome Archive (EGA) (study number EGAS00001002440; data set ID: EGAB00000001219) in a MINISEQE-compliant format. The EGA allows exploration of data sets from genomic studies. Access to data sets must be approved by the specified Data Access Committee through the European Molecular Biology Laboratory-European Bioinformatics Institute (EMBLEBI). The terms of use reflect EMBL-EBI's commitment to open science through its mission to provide freely available online services, databases, and software relating to data contributed from life science experiments to the largest possible community.

Plasmid transfection. For adherent cells, Lipofectamine 3000 (Invitrogen) was used according to the manufacturer's instructions. For DT40 cells, electroporation by Neon (Invitrogen) at 1,500 V, 20 ms, 1 pulse, was used.

Transduction. Generation of stable lentiviral producer lines and transduction of adherent cells were performed following the instructions in the Lenti-X Lentiviral Expression System User Manual (Takara Clontech). Because of inappropriate restriction sites, we modified the multiple cloning site using a double-stranded, sequence-verified genomic block (gBlocks Genomic Fragments, Integrated DNA Technologies). Its sequence is shown in Supplemental Table 5. The control vector pLVX-EF1 $\alpha$, expressing an IRES-puro cassette, and the RFWD3 vector PLVX-EF1 $\alpha+$ WT-RFWD3, additionally expressing human WT-RFWD3 cDNA, were constructed using classical cloning methods (primer sequences for cloning are listed in Supplemental Table 5). The missense mutation c.1916T>A encoding the I639K substitution was introduced using the Q5 Site-Directed Mutagenesis Kit (New England Biolabs) according to the manufacturer's instructions. Transduced cells were selected in puromycin (InvivoGen) for 7-14 days and challenged with MMC. We analyzed transduced fibroblasts and other cells by cell cycle analysis, did survival assays, or used other readout systems.

DT40 gene targeting. DT40 cells were cultured and $\triangle$ RFWD3 DT40 cells were generated by gene targeting as previously described (46). Briefly, a targeting vector with a histidinol or puromycin resistance cassette was used to substitute an approximately 1.2-kb genomic segment between chRFWD3 exons 2 and 3, similarly as reported elsewhere (25). The strategy is shown in Supplemental Figure 2A. Gene targeting replaced an approximately 9.3-kb WT genomic with an approximately $4.5-\mathrm{kb}$ targeted SacI fragment on Southern blots. chRFWD3 
cDNA was reverse transcribed from DT40 total RNA, cloned into pENTR plasmids, and transferred to expression plasmids using the Gateway system (Thermo Fisher Scientific). Generation of chRFW3 mutants was performed by QuikChange Site-Directed Mutagenesis Kit (Agilent Technologies) according to the manufacturer's instructions. To obtain stably expressing clones, chRFWD3-IRES-GFP- or GFPchRFWD3-expressing plasmids were transfected into $\triangle$ RFWD3 DT40 cells. Clones were selected by measurement of GFP expression levels on a FACSCalibur (Becton Dickinson) flow cytometer $(25,47)$.

Cell cycle, cell survival, and chromosomal studies. Flow cytometric cell cycle analysis was deployed as described after exposure of fibroblasts to $30 \mathrm{nM}$ (10 ng/ml) MMC (Sigma-Aldrich) or to $116 \mathrm{nM} \mathrm{(10} \mathrm{ng/}$ $\mathrm{ml}$ ) DEB (Acros Organics) for 48 hours $(48,49)$. For cell survival assays we adopted protocols reported by Kim et al. (50). Human fibroblasts were exposed to $20-100 \mathrm{nM}$ MMC or 0.1-16 $\mu \mathrm{M}$ cisplatin (Hexal) for 8 days. In knockdown studies, HAP1 cells were treated with FANCD2 siRNA and exposed to the indicated concentrations of MMC for 16 hours. After 8 hours of recovery, cells were plated with serial dilution, and colonies were counted after 7 days. To study MMC sensitivity of DT40 cells, they were serially diluted, exposed to an MMC pulse for 1 hour, and plated into medium containing 1.5\% methylcellulose. Colonies were counted after incubation for 1-2 weeks. Cell numbers were determined using a Nucleo Counter NC-250 (ChemoMetec). Assays were performed according to the manufacturer's protocols. To assess cisplatin sensitivity, DT40 cells were treated with the indicated concentration for 48 hours, and cell viability was assessed in solution using a FACSCalibur instrument after propidium iodide staining (46). For cell survival assays the commercially available DNA-topoisomerase I inhibitor camptothecin (Selleckchem) and the PARP inhibitor olaparib (Selleckchem) were used.

Human chromosome preparations were made by the air-drying method. Solid-stained metaphases were examined for chromatid- and chromosome-type damage. Whole-blood cultures were stimulated by phytohemagglutinin. They were exposed to MMC at final concentrations of 0,50 , or $100 \mathrm{ng} / \mathrm{ml}$ for 72 hours. Mitotic cells were arrested in metaphase using colcemid for 45 minutes. $0.075 \mathrm{M} \mathrm{KCl}$ was used as hypotonic treatment. Metaphase spreads were stained with $5 \%$ Giemsa solution. Slides were analyzed on an Axioskop Imager A1 (Zeiss) and scored for chromosomal instability. Cultured fibroblasts were processed similarly, but exposure to MMC was for 48 hours and exposure to colcemid for 2.5 hours, and $0.8 \mathrm{M}$ sodium citrate dihydrate was used as hypotonic treatment. Analysis of chromosome aberrations in DT40 cells was performed as previously described (25).

Immunoblotting and protein fractionation. Whole protein lysates of lymphoblasts or fibroblasts were obtained using Pierce IP Lysis Buffer (Thermo Fisher Scientific). Aliquots equaling $40 \mu \mathrm{g}$ of protein were prepared in $4 \times$ LDS Sample Buffer (Invitrogen), and 10× Sample Reducing Agent (Invitrogen) was added. Samples were heated to $70^{\circ} \mathrm{C}$ for 10 minutes and separated on $7 \%, 4 \%-12 \%$, or $3 \%-8 \%$ Bis-Tris gels by SDS-PAGE (Invitrogen). Proteins were transferred to a nitrocellulose membrane using the iBlot2 system (Invitrogen). After blocking of unspecific binding sites by $5 \%$ skim milk in PBS containing $0.1 \%$ Tween-20 (T) for 1 hour, antibodies listed in Supplemental Table 6 were applied. After 4 rounds of washing with PBS-T for 15 minutes, membranes were overlaid with Immobilon Western Chemiluminescent HRP Substrate (Millipore) and analyzed using the MicroChemi System (Berthold). Protein fractionation was achieved using the Sub- cellular Protein Fractionation Kit (Thermo Fisher Scientific) for cultured cells according to the manufacturer's instructions.

To isolate the chromatin fraction, DT40 cells were lysed in buffer A (10 mM HEPES, $10 \mathrm{mM} \mathrm{KCl,} 1.5 \mathrm{mM} \mathrm{MgCl}, 0.34 \mathrm{M}$ sucrose, $10 \%$ glycerol, 0.1\% Triton X-100 containing $1 \mathrm{mM}$ DTT, $1 \mathrm{mM} \mathrm{Na} \mathrm{VO}_{4}$, and protease inhibitor cocktail [Roche]). After incubation on ice for 30 minutes, samples were centrifuged at $1,700 \mathrm{~g}$ for 5 minutes. The pellets were washed with buffer A once. They were lysed in buffer $B$ (3 mM EDTA, $0.3 \mathrm{mM}$ EGTA, containing $1 \mathrm{mM}$ DTT, $1 \mathrm{mM} \mathrm{Na} \mathrm{VO}_{4}$, and protease inhibitor cocktail) and incubated on ice for $30 \mathrm{~min}$ utes. After centrifugation at 5,000 $g$ for 5 minutes, pellets contained the chromatin fraction. Proteins were separated by SDS-PAGE and detected by immunoblotting using, as indicated, anti-GFP (MBL International), anti-histone H3 (Active Motif) with the ECL prime kit, or Ponceau S staining (Ponceau S Solution, AppliChem).

CRISPR/Cas9 gene targeting. U2OS cells seeded in 6-well plates with $70 \%-80 \%$ confluence were transfected with pSPCas9(BB)2A-Puro (PX459, Addgene) using Lipofectamine 3000 (Life Technologies) according to the manufacturer's manual. Each transfection contained 3 different single guide RNAs (sgRNAs), generated using http:// crispr.mit.edu:8079/ (51). sgRNAs for targeting the RFWD3 gene were as follows: CR1, GGCACTGTCTTGTGACCTACAG; CR2, GCACTGTCTTGTGACCTACA; CR3, GGGGGCTGCATAGACTTTCA. All 3 sgRNAs are directed to exon 11; one of them even covers position c.1916 of the missense mutation. The same experiment was performed by cotransfection of U2OS cells with 1 of the sgRNAs and a template vector [pBlueScript II SK(+), Addgene] containing RFWD3 cDNA with c.1916T $>$ A. This vector was generated by classical cloning via the restriction sites SacI-HF and EcoRI-HF. Seventy-two hours after transfection, the medium was changed and selection with puromycin was launched for 4 days. Single cells were seeded in 96-well plates and expanded until sufficient cells were available for DNA isolation. Clones were analyzed by Sanger sequencing.

Immunofluorescence. Cells were grown on glass slides. Subconfluent cultures were exposed to $40 \mathrm{ng} / \mathrm{ml}$ of MMC and analyzed after indicated intervals as described previously (36). For U2OS experiments, cells were fixed with PBS containing 3\% paraformaldehyde, $2 \%$ sucrose, and $0.5 \%$ Triton $\mathrm{X}-100$ on ice for 30 minutes, and were then permeabilized with $0.5 \%$ Triton X-100/PBS for 5 minutes. After blocking with $2 \%$ BSA/PBS, samples were stained with antibodies diluted in $2 \%$ BSA/PBS for 1 hour at room temperature. The secondary antibodies used were Alexa Fluor 488-conjugated anti-mouse IgG or Alexa Fluor 594-conjugated anti-rabbit IgG (Molecular Probes). For DT40 experiments, cells were exposed to $500 \mathrm{ng} / \mathrm{ml} \mathrm{MMC} \mathrm{for} 8$ hours. DT40 cells were immobilized on glass slides using a Cytospin (Thermo Fisher Scientific) centrifuge, and were fixed with $4 \%$ paraformaldehyde for 30 minutes. DT40 cells were permeabilized with PBS containing $0.5 \%$ Triton $\mathrm{X}$ for 5 minutes and incubated with antiGFP monoclonal antibody (MBL International) or other antibodies at $37^{\circ} \mathrm{C}$ for 30 minutes. Nuclei were counterstained with DAPI, and slides were analyzed on a BIOREVO BZ-9000 microscope (Keyence).

RNA interference. Cells were transfected with siRNA using Lipofectamine RNAiMAX (Invitrogen) according to the manufacturer's instructions. Briefly, a dilution containing siRNA (10 nM) and Lipofectamine was premade using Opti-MEM I 1' (GIBCO) and then added to the cells. Four hours later the medium was replaced with fresh medium. Cells were allowed to grow for 24 hours before siRNA 
transfection was repeated. The following siRNAs were used: luciferase (5'-CGUACGCGGAAUACUUCGA-3'), BRCA2 (5'-GGAUUAUACAUAUUUCGCA-3'), RFWD3 (5'-GGACCUACUUGCAAACUAU-3'/ 5'-AUAGUUUGCAAGUAGGUCC-3'), and FANCD2 (5'-UAGAGAGUGAAGCAAACUCUG-3'). siLuciferase (siLuc) was used as a negative control in the RNA interference assays.

I-SceI-induced HR assay. Immortalized patient fibroblasts (cell line 1143) were stably transfected with a plasmid containing a WT copy of RFWD3 full-length cDNA (1143+WT), an empty vector (1143+mock), or a plasmid containing RFWD3 with the patient mutation (1143+I639K). The HR assay was performed in these cell lines by transient transfection of the pDR-GFP plasmid. Two days later, the cells were cotransfected with an I-SceI endonuclease expression vector (pCBASce), with an empty vector (pCAGGS), or with a plasmid constitutively expressing GFP (pNZE-GFP). In U2OS cells, the HR assay was performed as previously described (22). Briefly, U2OS cells were stably transfected with a vector containing a single copy of the DR-GFP construct and were transduced with specific siRNAs against luciferase, BRCA2, or RFWD3. Two days later, the cells were transfected with pCBASce, pCAGGS, or pNZE-GFP. In all cases, $2 \mu \mathrm{g}$ of plasmid was added in 6-well plates. Forty-eight hours after transfection, cells were trypsinized and resuspended in a buffer containing sodium citrate $(3.4 \mathrm{mM})$, Triton $(0.1 \%)$, RNase $(200 \mu \mathrm{g} / \mathrm{ml})$, and FBS $(20 \%)$. The number of green fluorescent cells was counted in a flow cytometer. Cells were finally stained with propidium iodide $(50 \mu \mathrm{g} /$ $\mathrm{ml}$ ), and the cell cycle distribution was determined by flow cytometry. The level of HR was quantified as the proportion of green fluorescent cells corrected by transfection rate and size of S phase. Plasmids for the I-SceI-induced HR assay were provided by Maria Jasin, Memorial Sloan Kettering Cancer Center, New York, New York, USA.

The same assay was performed in DT40 cells as previously described (25). We generated $\triangle$ RFWD3 DT40 and chRFWD3complemented $\triangle$ RFWD3 DT40 cell lines in which the SCneo construct with an I-SceI recognition site was targeted into the ovalbumin locus. To determine the nature of repair events, G418-selected colonies from WT and $\triangle$ RFWD3 cells were expanded, and genomic DNA was analyzed by long-range PCR as shown in Supplemental Figure 2E.

Coimmunoprecipitation. Cells were washed once with PBS, lysed in NETN buffer (150 mM NaCl, 0.5 mM EDTA, 20 mM Tris-HCl pH 8.0, $0.5 \% \mathrm{NP}-40$ ) supplemented with protease inhibitor cocktail, 25 $\mathrm{U} / \mathrm{ml}$ benzonase (Millipore), and $1 \mathrm{mM} \mathrm{Na}_{3} \mathrm{VO}_{4}$ on ice for 30 minutes, and centrifuged at approximately $16,000 \mathrm{~g}$ for 10 minutes. The pellet was discarded. Dynabeads Protein G (Invitrogen) was incubated with an appropriate primary antibody before it was added to the supernatant, following the manufacturer's instructions. To capture his-tagged or FLAG-tagged proteins, Complete His-Tag Purification Resin (Roche) or anti-FLAG M2 Agarose Affinity Gel (Sigma-Aldrich) was used, respectively. Captured proteins were washed and analyzed by immunoblotting. Samples were separated by SDS-PAGE and transferred to a PVDF membrane (Millipore). Proteins were detected as described previously (52).

Vector construction and targeting of mouse ES cells. A replacement vector for targeting of $R$ fw 3 exon 3 was generated by assembly of 3 fragments using sequential cloning. The final recipient vector contained the FRT-flanked neomycin selection (neo) cassette and the 3'-loxP site. PCR primers used to amplify the fragments included all the restriction enzyme sites required to join them together and to ligate them into the Surf2 vector backbone (Ozgene). The first fragment encompassed the 4.8-kb 5'-homology arm. The second fragment comprised the 0.7-kb region of exon 3 and was amplified using a sense primer (1589_42) that contained the 5'-loxP site. The third fragment encompassed the 3.6-kb 3'-homology arm. All of the fragments were amplified from BACs RP23-55G21 and RP23-297L3. For sequence information of the primers see Supplemental Table 7. The final targeting vector 1589_pTV contained the 5'-homology arm, exon 3 of Rfwd3, a neo cassette, and the 3'-homology arm (Supplemental Figure 3A). A PshAI and a ScaI site to be used for screening were inserted immediately $5^{\prime}$ of the $5^{\prime}$-loxP site and immediately $3^{\prime}$ of the $3^{\prime}$-loxP site, respectively. The FRT sites for conditional KO were not used. 1589_pTV was linearized by digestion with PmeI before electroporation into C57BL/6 Bruce 4 ES cells (29). Neo-resistant ES cell clones were screened by Southern hybridization to identify potentially targeted clones, using a probe downstream of the 3'-homology arm (P3). The WT allele showed a $6.5-\mathrm{kb}$ band and the correctly targeted allele a $4.1-\mathrm{kb}$ band when genomic DNA was digested with ScaI. Correct 5 '-integration was confirmed using a probe upstream of the $5^{\prime}$-homology arm (5Pi). The WT allele showed an 11.8-kb band and the correctly targeted allele a 5.3-kb band when genomic DNA was digested with PshAI. The possibility of additional random targeting events was precluded by use of a probe to the neo cassette (NeoP). Correctly targeted alleles showed a band size of $8.3 \mathrm{~kb}$ when genomic DNA was digested with PshAI. The KO allele was distinguished by Southern hybridization using probe P3. The WT allele showed a band size of $11.8 \mathrm{~kb}$, the targeted allele of $8.3 \mathrm{~kb}$, and the KO allele of $5.9 \mathrm{~kb}$ when genomic DNA was digested with PshAI.

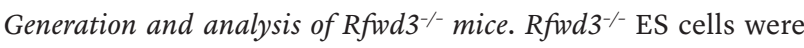
injected into goGermline blastocysts (30). Male chimeric mice were obtained and crossed to a ubiquitous Cre mouse line to remove the loxP-flanked exon 3. Eight- to ten-week-old $R$ fw $d 3^{-/-}$mice were generated from heterozygous breeding. All strains were backcrossed on a C57BL/ 6 background for more than 3 generations. Mouse embryonic fibroblasts (MEFs) were obtained from embryos of $R$ fw $d 3^{-/-}$ male mice crossed with $R$ fw $d^{+/-}$females. Genotyping of the pups confirmed their Rfivd3 status (Supplemental Figure 3B). Phenotypical analyses of homozygous $R$ fiw $d 3^{-/-}$mice were performed at Ozgene by a veterinarian and according to ethical guidelines of Australia. Blood analyses were done by cutting of the tail vein. All experiments in mice were performed in accordance with protocols approved by the Ozgene Animal Ethics Committee under the proposal 150602 PHEN, project 1589_Cajun.

Examination of MEFs. MEFs were cultured in DMEM (SigmaAldrich) with 10\% FBS (Sigma-Aldrich), 0.2\% $\beta$-mercaptoethanol (Life Technologies), and $2 \mathrm{mM}$ GlutaMax (Gibco). Survival assays, chromosomal breakage, and flow cytometric cell cycle analyses were performed similarly to experiments with other cell types. The types of mice used in this study are included in Supplemental Table 3.

Real-time PCR analysis of Rfiwd3 mRNA expression. Liver samples were harvested, snap-frozen in liquid nitrogen, and stored at $-80^{\circ} \mathrm{C}$. mRNA was extracted from frozen tissue using the PureLink RNA Mini Kit (Thermo Fisher Scientific) per supplied protocol. DNase digestion on column was included. Extracted mRNA was reverse transcribed using the High-Capacity cDNA Reverse Transcription Kit (Thermo Fisher Scientific) per supplied protocol. Two quantitative PCR (qPCR) assays were designed to detect the regions of exons 3-5 and of exons 6-8. Real-time PCR was performed using the CFX Connect Real-Time 
PCR Detection System (Bio-Rad). Each duplex PCR reaction consisted of $0.5 \mu \mathrm{l} 40^{\prime} \beta$-actin qPCR assay, $1 \mu \mathrm{l} 20^{\prime}$ Rfiwd3 qPCR assay, $1 \mu \mathrm{l}$ cDNA, $7.5 \mu \mathrm{l} \mathrm{H}_{2} \mathrm{O}$, and $10 \mu \mathrm{l}$ 2' PrimeTime Gene Expression Master Mix (Integrated DNA Technologies, IDT) in a $20-\mu$ l reaction per IDT assay protocols. This resulted in a final $1 \times$ concentration of $250 \mathrm{nM}$ for each of the probes, and of $500 \mathrm{nM}$ for each primer set. Sequence information of the Rfivd3 primers and probes used in these assays is shown in Supplemental Table 7. Amplification conditions were $95^{\circ} \mathrm{C}$ for 3 minutes followed by 40 cycles of 15 seconds denaturation at $95^{\circ} \mathrm{C}$ and 1 minute annealing at $62^{\circ} \mathrm{C}$. All qPCR reactions on cDNA were carried out in triplicate with "no template" and "no reverse transcriptase" control samples. Data were analyzed using CFX Manager Software, version 3.1 (Bio-Rad). Relative expression of Rfwd3 mRNA in $R f w d 3^{-/-}$and $R f w d 3^{+/+}$samples was calculated by the $\Delta \Delta C T$ method.

Statistics. For the siRNA or other transfection assays in U2OS or DT40 cells and immunofluorescence analysis of 1143, 1143 WTRFWD3-complemented, and non-FA cells, 3 independent experiments each were done, and the 2-tailed Student's $t$ test was used to compare the results. With patient-derived fibroblasts, 5 independent experiments were done, and the 2-tailed Student's $t$ test or the MannWhitney test was performed depending on whether the data were normally distributed. For the epistasis study (Figure 4H), 3 independent experiments each were done, and the Tukey's range test was used to compare the results (53). Statistical analyses were performed using SPSS software. A $P$ value less than 0.05 was considered significant; ${ }^{*} P<0.05,{ }^{* *} P<0.01,{ }^{* *} P<0.001$.

Study approval. The parents of the patient gave their informed consent to patient-related studies. Genetic work on FA was approved by the Institutional Review Board of the Faculty of Medicine at the University of Wurzburg. FANCW being used as an alias for RFWD3 has been approved by HUGO Gene Nomenclature Committee (HGNC).

\section{Author contributions}

KK designed and performed experiments, analyzed data, prepared the figures, and wrote the manuscript. SI designed and performed DT40 and HAP1 cell studies with MI, analyzed DT40 data, contributed results, and wrote parts of the manuscript. MJR and JS performed the I-SceI-induced HR assay with human cell lines, analyzed the data, contributed results, and wrote parts of the manuscript. MT and DS conceived the experiments, analyzed data, contributed results, supervised the study, and wrote the manuscript.

\section{Acknowledgments}

We are grateful to the patient and her family for providing information on the disease course and agreeing to publication in an anonymized manner. We thank the attending pediatrician, A. Gnekow, for medical information and great cooperation. We deeply appreciate expert technical contributions by R. Friedl. We also express our gratitude to J. Jardine for sharing mouse histology data and to R. Kalb for discussions, figure preparation, and critical reading of the manuscript. This work was supported, in part, by a grant from the Schroeder-Kurth Fund (to KK and DS) and by Japan Society for the Promotion of Science (JSPS) KAKENHI grants JP23114010 (to MT), JP26550026 (to MT), JP15H01738 (to MT), and JP26281021 (to MI). The Radiation Biology Center is a Joint Usage Research Center supported by the Ministry of Education, Culture, Sports, Science and Technology of Japan.

Address correspondence to: Detlev Schindler, University of Wurzburg, Department of Human Genetics, Biozentrum, Am Hubland, D-97074 Wurzburg, Germany. Phone: 49.931.318.4089; Email: schindler@biozentrum.uni-wuerzburg.de.
1. Kutler DI, et al. High incidence of head and neck squamous cell carcinoma in patients with Fanconi anemia. Arch Otolaryngol Head Neck Surg. 2003;129(1):106-112.

2. Auerbach AD. Fanconi anemia and its diagnosis. Mutat Res. 2009;668(1-2):4-10.

3. Kutler DI, et al. Natural history and management of Fanconi anemia patients with head and neck cancer: A 10-year follow-up. Laryngoscope. 2016;126(4):870-879.

4. Alter BP. Fanconi anemia and the development of leukemia. Best Pract Res Clin Haematol. 2014;27(3-4):214-221.

5. Kottemann MC, Smogorzewska A. Fanconi anaemia and the repair of Watson and Crick DNA crosslinks. Nature. 2013;493(7432):356-363.

6. Wang AT, Smogorzewska A. SnapShot: Fanconi anemia and associated proteins. Cell. 2015;160(1-2):354-354.e1.

7. Schwertman P, Bekker-Jensen S, Mailand N. Regulation of DNA double-strand break repair by ubiquitin and ubiquitin-like modifiers. Nat Rev Mol Cell Biol. 2016;17(6):379-394.

8. Bluteau D, et al. Biallelic inactivation of REV7 is associated with Fanconi anemia. JClin Invest. 2016;126(9):3580-3584.

9. Ceccaldi R, Sarangi P, D’Andrea AD. The Fanconi anaemia pathway: new players and new functions. Nat Rev Mol Cell Biol. 2016;17(6):337-349.
10. Moldovan GL, D'Andrea AD. How the fanconi anemia pathway guards the genome. Annu Rev Genet. 2009;43:223-249.

11. Hershko A, Ciechanover A. The ubiquitin system. Annu Rev Biochem. 1998;67:425-479.

12. Murai J, Yang K, Dejsuphong D, Hirota K, Takeda S, D'Andrea AD. The USP1/UAF1 complex promotes double-strand break repair through homologous recombination. Mol Cell Biol. 2011;31(12):2462-2469.

13. Paquin KL, Vierra DA, Howlett NG. A DUBless step? Tighten up D-loop. Cell Cycle. 2016;15(23):3163-3164.

14. Elia AE, et al. RFWD3-dependent ubiquitination of RPA regulates repair at stalled replication forks. Mol Cell. 2015;60(2):280-293.

15. Matsuoka S, et al. ATM and ATR substrate analysis reveals extensive protein networks responsive to DNA damage. Science. 2007;316(5828):1160-1166.

16. Fu X, et al. RFWD3-Mdm2 ubiquitin ligase complex positively regulates $\mathrm{p} 53$ stability in response to DNA damage. Proc Natl Acad Sci U S A. 2010;107(10):4579-4584.

17. Gong Z, Chen J. E3 ligase RFWD3 participates in replication checkpoint control. J Biol Chem. 2011;286(25):22308-22313.

18. Liu S, et al. RING finger and WD repeat domain 3 (RFWD3) associates with replication protein A (RPA) and facilitates RPA-mediated DNA damage response. J Biol Chem. 2011;286(25):22314-22322.

19. Wang AT, et al. A dominant mutation in human RAD51 reveals its function in DNA interstrand crosslink repair independent of homologous recombination. Mol Cell. 2015;59(3):478-490.

20. Ameziane N, et al. A novel Fanconi anaemia subtype associated with a dominant-negative mutation in RAD51. Nat Commun. 2015;6:8829.

21. Stivaros SM, et al. Central nervous system abnormalities in Fanconi anaemia: patterns and frequency on magnetic resonance imaging. $\mathrm{Br} \mathrm{J}$ Radiol. 2015;88(1056):20150088.

22. Zhang F, Shi J, Bian C, Yu X. Poly(ADP-Ribose) mediates the BRCA2-dependent early DNA damage response. Cell Rep. 2015;13(4):678-689.

23. Jasin M, Rothstein R. Repair of strand breaks by homologous recombination. Cold Spring Harb Perspect Biol. 2013;5(11):a012740.

24. Pierce AJ, Johnson RD, Thompson LH, Jasin M. XRCC3 promotes homology-directed repair of DNA damage in mammalian cells. Genes Dev. 1999;13(20):2633-2638.

25. Yamamoto K, et al. Fanconi anemia FANCG protein in mitigating radiation- and enzyme-induced DNA double-strand breaks by homologous recombination in vertebrate cells. Mol Cell Biol. 2003;23(15):5421-5430.

26. Takata M, et al. Chromosome instability and defective recombinational repair in knockout 
mutants of the five Rad51 paralogs. Mol Cell Biol. 2001;21(8):2858-2866.

27. Kitao H, Yamamoto K, Matsushita N, Ohzeki M, Ishiai M, Takata M. Functional interplay between BRCA2/FancD1 and FancC in DNA repair. J Biol Chem. 2006;281(30):21312-21320.

28. Wang X, Andreassen PR, D'Andrea AD. Functional interaction of monoubiquitinated FANCD2 and BRCA2/FANCD1 in chromatin. Mol Cell Biol. 2004;24(13):5850-5862.

29. Köntgen F, Süss G, Stewart C, Steinmetz M, Bluethmann H. Targeted disruption of the MHC class II Aa gene in C57BL/6 mice. Int Immunol. 1993;5(8):957-964.

30. Koentgen F, et al. Exclusive transmission of the embryonic stem cell-derived genome through the mouse germline. Genesis. 2016;54(6):326-333.

31. Gong Z, Chen J. E3 ligase RFWD3 participates in replication checkpoint control. J Biol Chem. 2011;286(25):22308-22313.

32. Park JY, et al. Complementation of hypersensitivity to DNA interstrand crosslinking agents demonstrates that XRCC2 is a Fanconi anaemia gene. JMed Genet. 2016;53(10):672-680.

33. Yokoyama $\mathrm{H}$, et al. Preferential binding to branched DNA strands and strand-annealing activity of the human Rad51B, Rad51C, Rad51D and Xrcc2 protein complex. Nucleic Acids Res. 2004;32(8):2556-2565.

34. Howlett NG, et al. Biallelic inactivation of BRCA2 in Fanconi anemia. Science. 2002;297(5581):606-609.
35. Reid S, et al. Biallelic mutations in PALB2 cause Fanconi anemia subtype FA-N and predispose to childhood cancer. Nat Genet. 2007;39(2):162-164.

36. Vaz F, et al. Mutation of the RAD51C gene in a Fanconi anemia-like disorder. Nat Genet. 2010;42(5):406-409.

37. Shamseldin HE, Elfaki M, Alkuraya FS. Exome sequencing reveals a novel Fanconi group defined by XRCC 2 mutation. JMed Genet. 2012;49(3):184-186.

38. Sawyer SL, et al. Biallelic mutations in BRCA1 cause a new Fanconi anemia subtype. Cancer Discov. 2015;5(2):135-142.

39. Xu Y, Her C. Inhibition of Topoisomerase (DNA) I (TOP1): DNA damage repair and anticancer therapy. Biomolecules. 2015;5(3):1652-1670.

40. Murai J, et al. Trapping of PARP1 and PARP2 by clinical PARP inhibitors. Cancer Res. 2012;72(21):5588-5599.

41. Dong H, Nebert DW, Bruford EA, Thompson DC, Joenje H, Vasiliou V. Update of the human and mouse Fanconi anemia genes. Hum Genomics. 2015;9:32.

42. Crossan GP, et al. Disruption of mouse Slx 4 , a regulator of structure-specific nucleases, phenocopies Fanconi anemia. Nat Genet. 2011;43(2):147-152.

43. Bakker ST, de Winter JP, te Riele H. Learning from a paradox: recent insights into Fanconi anaemia through studying mouse models. Dis Model Mech. 2013;6(1):40-47.

44. Katsuki Y, Takata M. Defects in homologous recom- bination repair behind the human diseases: FA and HBOC. Endocr Relat Cancer. 2016;23(10):T19-T37.

45. Knies K, et al. Genotyping of fanconi anemia patients by whole exome sequencing: advantages and challenges. PLoS One. 2012;7(12):e52648.

46. Ishiai M, et al. FANCI phosphorylation functions as a molecular switch to turn on the Fanconi anemia pathway. Nat Struct Mol Biol. 2008;15(11):1138-1146.

47. Nishimura K, et al. Mcm8 and Mcm9 form a complex that functions in homologous recombination repair induced by DNA interstrand crosslinks. Mol Cell. 2012;47(4):511-522.

48. Seyschab H, et al. Comparative evaluation of diepoxybutane sensitivity and cell cycle blockage in the diagnosis of Fanconi anemia. Blood. 1995;85(8):2233-2237.

49. Auerbach AD. Fanconi anemia diagnosis and the diepoxybutane (DEB) test. Exp Hematol. 1993;21(6):731-733.

50. Kim Y, Spitz GS, Veturi U, Lach FP, Auerbach AD, Smogorzewska A. Regulation of multiple DNA repair pathways by the Fanconi anemia protein SLX4. Blood. 2013;121(1):54-63.

51. Ran FA, Hsu PD, Wright J, Agarwala V, Scott DA, Zhang F. Genome engineering using the CRISPRCas9 system. Nat Protoc. 2013;8(11):2281-2308.

52. Unno J, et al. FANCD2 binds CtIP and regulates DNA-end resection during DNA interstrand crosslink repair. Cell Rep. 2014;7(4):1039-1047.

53. Tukey JW. Comparing individual means in the analysis of variance. Biometrics. 1949;5(2):99-114. 